TRANSACTIONS OF THE

AMERICAN MATHEMATICAL SOCIETY

Volume 361, Number 11, November 2009, Pages 5781-5803

S 0002-9947(09)04760-6

Article electronically published on June 4, 2009

\title{
STRINGY PRODUCT ON TWISTED ORBIFOLD K-THEORY FOR ABELIAN QUOTIENTS
}

\author{
EDWARD BECERRA AND BERNARDO URIBE
}

\begin{abstract}
In this paper we present a model to calculate the stringy product on twisted orbifold K-theory of Adem-Ruan-Zhang for abelian complex orbifolds.

In the first part we consider the non-twisted case on an orbifold presented as the quotient of a manifold acted by a compact abelian Lie group. We give an explicit description of the obstruction bundle, we explain the relation with the product defined by Jarvis-Kaufmann-Kimura and, via a Chern character map, with the Chen-Ruan cohomology, we explicitly calculate the stringy product for a weighted projective orbifold.

In the second part we consider orbifolds presented as the quotient of a manifold acted by a finite abelian group and twistings coming from the group cohomology. We show a decomposition formula for twisted orbifold K-theory that is suited to calculate the stringy product and we use this formula to calculate two examples when the group is $(\mathbb{Z} / 2)^{3}$.
\end{abstract}

\section{INTRODUCTION}

This paper is devoted to the study of the stringy product on the twisted orbifold K-theory defined by Adem-Ruan-Zhang ARZ.

In the first part we consider the non-twisted case on an orbifold presented as the quotient of a manifold acted by a compact abelian Lie group. By means of methods used to understand the obstruction bundle developed in [CH06, JKK07, we were able to give a very explicit description of the obstruction bundle in terms of $G$-equivariant bundles. With this model in hand, the calculation of the obstruction bundle becomes a simple diagonalization procedure and a projection onto some of the components. One of the key advantages of this construction is that everything can be performed without the use of rational coefficients, and therefore the torsion classes of equivariant K-theory are not disregarded.

We construct a stringy ring on the K-theory of the Borel construction of the inertia orbifold, similar to the stringy structure in K-theory that was defined in JKK07. This construction does not need rational coefficients either, and it uses the same principle of the stringy product.

Received by the editors June 27, 2007.

2000 Mathematics Subject Classification. Primary 14N35, 19L47; Secondary 55N15, 55N91.

Key words and phrases. Stringy product, twisted orbifold K-theory, Chen-Ruan cohomology, inverse transgression map.

Both authors acknowledge the support of COLCIENCIAS through the grant 120440520246 and of CONACYT-COLCIENCIAS throught contract number 376-2007.

The second author was partially supported by the "Fondo de apoyo a investigadores jovenes" from Universidad de los Andes.

(C)2009 American Mathematical Society Reverts to public domain 28 years from publication 
Applying a calibrated Chern character map (mainly due to Jarvis-KaufmannKimura [JKK07]), we were able to prove that there is a ring homomorphism between the stringy ring on the K-theory of the inertia orbifold and the Chen-Ruan cohomology of the orbifold. We come to the conclusion that the right place to study stringy products is K-theory and not cohomology; this is due to the fact that the torsion classes in the stringy K-theory are present.

We calculate an explicit example of the rings that we have defined in the case of a weighted projective orbifold, and for doing so, we make use of a decomposition of equivariant K-theory developed in AR03.

In the second part we consider orbifolds presented as the quotient of a manifold acted by a finite abelian group and twistings coming from the group cohomology. We recall the definition of the twisting classes via the inverse transgression map and we give a simple argument to show that these classes must be torsion. We calculate the inverse transgression map in the case that the group is $(\mathbb{Z} / 2)^{3}$ and we expand on an example previously constructed in ARZ.

Then we show a decomposition formula for twisted orbifold K-theory that can be considered as a hybrid between the decomposition formula for equivariant Ktheory using cyclotomic fields and the decomposition for twisted orbifold K-theory (both in [AR03]). We finish the paper using this formula to calculate the stringy product in twisted orbifold K-theory with rational coefficients when the orbifolds are $\left[* /(\mathbb{Z} / 2)^{3}\right]$ and $\left[T^{6} /(\mathbb{Z} / 2)^{3}\right]$ and when the twisting classes are non-trivial.

It was pointed out to us by A. Adem that the decomposition formula is not necessary to calculate the stringy product in twisted orbifold K-theory in the case of $\left[* /(\mathbb{Z} / 2)^{3}\right]$ and that an explicit calculation of this ring was carried out by A. N. Duman in Dum.

\section{Stringy PRODUCT ON THE K-THEORY OF the INERTIA ORBIFOld}

Let $M$ be a compact manifold and $G$ a compact abelian Lie group that acts almost freely on $M$, such that the orbifold $[M / G]$ is endowed with an almost complex structure. In what follows we will define a stringy product on the equivariant $\mathrm{K}$ theory of the inertia orbifold of $[M / G]$ in as much the same way as the Chen-Ruan product in orbifold cohomology. We will show that our definition will be equivalent to the one defined in ARZ without twisting. The key ingredient of the definition will be the obstruction bundle and its construction will be outlined in this section.

Consider the set $\mathcal{C}=\left\{g \in G \mid M^{g} \neq \phi\right\}$ where $M^{g}$ denotes the fixed point set of the action of $g$. As $G$ is compact abelian and the action is almost free, the set $\mathcal{C}$ is finite.

In the case in which we are interested, the inertia orbifold (see [Moe02]) of $[M / G]$ is

$$
I[M / G]=\bigsqcup_{g \in \mathcal{C}}\left[M^{g} / G\right]
$$

where $G$ acts in the natural way.

Definition 2.1. The stringy K-theory of the inertia orbifold $K_{s t}([M / G])$, as a $\mathbb{Z}$-module, is defined as the K-theory of its inertia orbifold, i.e.

$$
K_{s t}^{*}([M / G]):=K^{*}(I[M / G]) \cong \bigoplus_{g \in \mathcal{C}} K_{G}^{*}\left(M^{g}\right) \times\{g\} .
$$


Note that the summands in the right hand side are in $G$-equivariant K-theory Seg68.

The ring structure of the stringy K-theory defined in ARZ uses the genus zero, three point function, Gromov-Witten invariants approach. In this paper we will show an equivalent description for this ring structure that has the advantage that it is very simple to use when one wants to calculate explicit examples.

2.1. Obstruction bundle. Let us start by noting that as the orbifold $[M / G]$ is almost complex; then the normal bundles of the fixed point sets $M^{g}$ are all $G$ equivariant complex bundles. Now, take $g, h \in \mathcal{C}$ and consider the space $M^{g, h}:=$ $M^{g} \cap M^{h}$. As $g$ and $h$ commute, one can simultaneously diagonalize the action of $g$ and $h$ on the normal bundle $V_{g, h}$ of the inclusion $M^{g, h} \rightarrow M$. Therefore, as the order of both $g$ and $h$ is finite, $V_{g, h}$ is a complex bundle isomorphic to $V_{1} \oplus \cdots \oplus V_{k}$ where $V_{j}$ is simultaneously the eigenbundle of $e^{2 \pi i a_{j}}$ for the action of $g$ and of $e^{2 \pi i b_{j}}$ for the action of $h$, with $a_{j}, b_{j} \in \mathbb{Q}$ and $0 \leq a_{j}, b_{j}<1$. Note that (and this is a key fact) as the group $G$ is abelian, all the bundles $V_{j}$ are $G$-equivariant (this construction is performed in each connected component of $M^{g, h}$ ).

Definition 2.2. The obstruction bundle $D_{g, h}$ for the pair $g, h$ is defined as

$$
D_{g, h}:=\bigoplus_{\left\{j \mid a_{j}+b_{j}>1\right\}} V_{j},
$$

and is a $G$-equivariant bundle over $M^{g, h}$.

Remark 2.3. If we take $k=(g h)^{-1}$ and $e^{2 \pi i c_{j}}$ as the eigenvalue of the action of $k$ in $V_{j}\left(0 \leq c_{j}<1\right)$, then we have also that

$$
D_{g, h}=\bigoplus_{\left\{j \mid a_{j}+b_{j}+c_{j}=2\right\}} V_{j} .
$$

This bundle is the obstruction bundle that is given in CH06. Prop. 1 page 65] for abelian orbifolds. We are adding the fact that this bundle is $G$-equivariant.

The obstruction bundle $D_{g, h}$ defined above is equivalent to the obstruction bundle given in [JKK07, Def. 1.5] in the case where the group $G$ is finite. Let's recall their definition.

For $g \in \mathcal{C}$ split the normal bundle $W_{g}$ of the inclusion $M^{g} \rightarrow M$ into eigenbundles of the $g$ action. Let $W_{g}^{l}$ be the eigenbundle of $e^{2 \pi i a_{l}}$ and define the bundle (in $\left.K^{0}\left(M^{g}\right) \otimes \mathbb{Q}\right)$

$$
\mathcal{F}_{g}:=\bigoplus_{l} a_{j} W_{g}^{l}
$$

The obstruction bundle $B_{g, h}$ of [JKK07] is

$$
B_{g, h}:=\left.\left.\left.\left.T M^{g, h} \ominus T M\right|_{M^{g, h}} \oplus \mathcal{F}_{g}\right|_{M^{g, h}} \oplus \mathcal{F}_{h}\right|_{M^{g, h}} \oplus \mathcal{F}_{(g h)^{-1}}\right|_{M^{g, h}} .
$$

Lemma 2.4. When the group $G$ is finite, the bundle $D_{g, h}$ and the virtual bundle $B_{g, h}$ represent the same class in $K^{0}\left(M^{g, h}\right) \otimes \mathbb{Q}$.

Proof. Let's write the virtual bundle $B_{g, h}$ in terms of the bundles $V_{j}$. By restriction we have that

$$
\left.\mathcal{F}_{g}\right|_{M^{g, h}} \cong \bigoplus_{j} a_{j} V_{j},\left.\quad \mathcal{F}_{h}\right|_{M^{g, h}} \cong \bigoplus_{j} b_{j} V_{j} \text { and }\left.\mathcal{F}_{(g h)^{-1}}\right|_{M^{g, h}} \cong \bigoplus_{j} c_{j} V_{j} .
$$


Then

$$
B_{g, h} \cong \bigoplus_{j} a_{j} V_{j} \oplus \bigoplus_{j} b_{j} V_{j} \oplus \bigoplus_{j} c_{j} V_{j} \ominus \bigoplus_{j} V_{j} \cong \bigoplus_{j}\left(a_{j}+b_{j}+c_{j}-1\right) V_{j}=D_{g, h}
$$

2.2. Stringy product on K-theory of the inertia orbifold. With the obstruction bundle in hand, we can now define the stringy product on the K-theory of the inertia orbifold. Let's denote the inclusions by

$$
e_{1}: M^{g, h} \rightarrow M^{g} \quad e_{2}: M^{g, h} \rightarrow M^{h} \quad e_{3}: M^{g, h} \rightarrow M^{g h} .
$$

Definition 2.5. For the almost complex orbifold $[M / G]$, define the stringy product $\star$ in the stringy $\mathrm{K}$-theory $K_{s t}^{*}([M / G])$ as follows:

$$
\begin{aligned}
\star:\left(K_{G}^{*}\left(M^{g}\right) \times\{g\}\right) \times\left(K_{G}^{*}\left(M^{h}\right) \times\{h\}\right) & \rightarrow K_{G}^{*}\left(M^{g h}\right) \times\{g h\}, \\
((F, g),(H, h)) & \mapsto\left(e_{3 !}\left(e_{1}^{*} F \otimes e_{2}^{*} H \otimes \lambda_{-1}\left(D_{g, h}\right)\right), g h\right),
\end{aligned}
$$

where $g, h \in \mathcal{C}$, the pullbacks and pushforwards are defined in the equivariant setup and $\lambda_{-1}\left(D_{g, h}\right)$ is the Euler class of the bundle $D_{g, h}$, i.e. the restriction to $M^{g, h}$ of the Thom class of $D_{g, h}$ (see [Seg68, Prop. 3.2]).

Proposition 2.6. The ring structure in $K_{s t}^{*}([M / G])$ defined above in Definition 2.5 is the same as the ring structure defined in Definition 7.3 of [ARZ].

Proof. By Remark 2.3 above we know that in Proposition 1 of [CH06] it is proved that the obstruction bundle of the Chen-Ruan cohomology is isomorphic to the obstruction bundle $D_{g, h}$. As in ARZ the obstruction bundle is the obstruction bundle of the Chen-Ruan cohomology [CR04; then the two ring structures agree.

2.3. Associativity. The fact that $K_{s t}^{*}([M / G])$ together with the stringy product in K-theory is associative is proved in Theorem 7.4 [ARZ]. It is basically the same proof that is given in lemma 5.4 of [JKK07] but in the equivariant setup. We are not going to reproduce the proof here because it would be redundant. Instead, we will write some words on the key ingredient of the proof which is the equivariant version of the excess intersection formula of Quillen.

In Proposition 3.3 of Qui71 it is shown that for the inclusion of almost complex manifolds

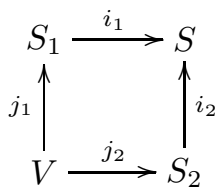

with $V=S_{1} \cap S_{2}$, then

$$
i_{2}^{*} i_{1 *} x=j_{2 *}\left(e\left(E\left(S, S_{1}, S_{2}\right)\right) \cdot j_{1}^{*} x\right),
$$

holds in cohomology where $e\left(E\left(S, S_{1}, S_{2}\right)\right.$ is the Euler class of the bundle

$$
E\left(S, S_{1}, S_{2}\right)=\left.\left.\left.T S\right|_{V} \oplus T V \ominus T S_{1}\right|_{V} \ominus T S_{2}\right|_{V} .
$$

This bundle is known as the excess intersection bundle.

The formula also holds in K-theory, namely

$$
i_{2}^{*} i_{1 !} F=j_{2 !}\left(\lambda_{-1}\left(E\left(S, S_{1}, S_{2}\right)\right) \otimes j_{1}^{*} F\right) .
$$


Both formulas rely on the fact that for a complex bundle $E \rightarrow V$ with zero section $\sigma: V \rightarrow E$, one has $\sigma^{*} \sigma_{*} x=e(E) \cdot x$ in cohomology and $\sigma^{*} \sigma_{!} F=\lambda_{-1}(E) \otimes F$ in K-theory.

In the case that the bundle $E \rightarrow V$ is equivariant, Segal showed that the formula $\sigma^{*} \sigma_{!} F=\lambda_{-1}(E) \otimes F$ also applies to the equivariant setup [Seg68, Page 140]; therefore the excess intersection formula of Quillen holds in the case where the manifolds $S, S_{1}, S_{2}, V$ and the maps $i_{i}, j_{1}, i_{2}, j_{2}$ are all equivariant.

With the equivariant excess intersection formula in hand, the associativity boils down to check the following equality in $K_{G}^{0}(N)$ where $N=M^{g, h, k}$ :

$\left.\left.D_{g, h}\right|_{N} \oplus D_{g h, k}\right|_{N} \oplus E\left(M^{g h}, M^{g, h}, M^{g h, k}\right)=\left.\left.D_{g, h k}\right|_{N} \oplus D_{h, k}\right|_{N} \oplus E\left(M^{h k}, M^{g, h k}, M^{h, k}\right)$.

The proof follows the same lines as the proof of lemma 5.4 of [JKK07]; we only need to remark that in our case all the operations are done in the $G$-equivariant setup. We will not reproduce the proof.

2.4. Weighted projective orbifold $\mathbb{C} P[p: 1: \cdots: 1]$. In this section we are going to calculate the stringy K-theory in the case of the weighted projective orbifold $\mathbb{C} P[p: 1: \cdots: 1]$. Let's recall its definition.

Let $M=S^{2 n+1}=\left\{\left.\left(z_{0}, \ldots, z_{n}\right) \in \mathbb{C}^{n+1}\left|\sum_{i}\right| z_{i}\right|^{2}=1\right\}$ and $G=S^{1}$ acting on $M$ as

$$
\begin{aligned}
S^{1} \times S^{2 n+1} & \rightarrow S^{2 n+1} \\
\left(\lambda,\left(z_{0}, \ldots, z_{n}\right)\right) & \mapsto\left(\lambda^{p} z_{0}, \lambda z_{1}, \lambda z_{2}, \ldots, \lambda z_{n}\right)
\end{aligned}
$$

with $p$ a prime number. The quotient space $M / G$ is the weighted projective space $\mathbb{C} P(p, 1, \ldots, 1)$, and the orbifold $[M / G]$ is the weighted projective orbifold $\mathbb{C} P[p: 1: \cdots: 1]$.

The set $\mathcal{C}$ is equal to the elements in the group $\mathbb{Z} / p \subset G$ which we will denote by $\left\{g_{0}=i d, g_{1}, \ldots, g_{p-1}\right\}=\mathcal{C}$. The fixed point sets are $M^{g_{0}}=M$ and $M^{g_{j}}=$ $\left\{\left(z_{0}, 0, \ldots, 0\right) \in M\right\}=S^{1}$ for $1 \leq j<p$. The $G$ action on $M^{g_{j}}$ is the same as the action of $G$ on the space $S^{1} /(\mathbb{Z} / p)$ given by rotation. Then as a $\mathbb{Z}$-module

$$
K_{s t}^{*}([M / G])=K_{G}^{*}\left(S^{2 n+1}\right) \times\left\{g_{0}\right\} \oplus \bigoplus_{j=1}^{p-1} K_{G}^{*}\left(S^{1} /(\mathbb{Z} / p)\right) \times\left\{g_{j}\right\} .
$$

As the action of $G$ in $M^{g_{j}} \cong S^{1} /(\mathbb{Z} / p)$ is by rotation, then we know that

$$
K_{G}^{*}\left(S^{1} /(\mathbb{Z} / p)\right) \cong K_{\mathbb{Z} / p}^{*}(p t) \cong R(\mathbb{Z} / p),
$$

where $R(\mathbb{Z} / p)$ is the Grothendieck ring of $\mathbb{Z} / p$ representations. Therefore, the stringy $\mathrm{K}$-theory, as a $\mathbb{Z}$-module, is isomorphic to

$$
K_{s t}^{*}([M / G])=K_{G}^{*}\left(S^{2 n+1}\right) \times\left\{g_{0}\right\} \oplus \bigoplus_{j=1}^{p-1} R(\mathbb{Z} / p) \times\left\{g_{j}\right\} .
$$

For $g_{j}, g_{k} \in \mathcal{C}, j \neq 0$, the obstruction bundle $D_{j, k}:=D_{g_{j}, g_{k}}$ is a subbundle of the normal bundle $V_{j, k}$ of the inclusion $S^{1} \rightarrow S^{2 n+1}$, where in this case $M^{g_{j}, g_{k}}=$ $M^{g_{j}}=S^{1}$. The normal bundle $V_{j, k}$ is isomorphic to the trivial bundle $S^{1} \times \mathbb{C}^{n}$ over $S^{1}$, where the circle action is given by $\lambda(\eta, \mathbf{z})=\left(\lambda^{p} \eta, \lambda \mathbf{z}\right)$. This follows from the fact that one can take the set

$$
\left\{\left(z_{0}, \ldots, z_{n}\right) \in S^{2 n+1}|| z_{i} \mid<\frac{1}{n} \text { for } 1 \leq i \leq n\right\}
$$


as a tubular neighborhood of the inclusion $S^{1} \rightarrow S^{2 n+1}$, and this set is isomorphic to

$$
S^{1} \times\left\{\left(z_{1}, \ldots, z_{n}\right) \in \mathbb{C}^{n}|| z_{i} \mid<\frac{1}{n}\right\}
$$

Then the normal bundle can be seen as $n W \in R(\mathbb{Z} / p) \cong K_{S^{1}}^{*}\left(M^{g_{j}, g_{k}}\right)$, where $W$ is the one dimensional irreducible representation whose character is $e^{\frac{2 \pi i}{p}}$.

Going back to the obstruction bundle, we have that

$$
D_{j, k}=\left\{\begin{array}{cl}
V_{j, k} & \text { for } \quad j+k>p \\
0 & \text { for } \quad j+k \leq p
\end{array}\right.
$$

This is because the action of $g_{j}$ on the normal bundle $V_{j, k}$ is given by multiplying by $e^{\frac{2 \pi i}{p} j}$.

Having defined the obstruction bundle, we need to explicitly determine the pullbacks and pushforwards of the inclusion maps. The first step is to calculate explicitly $K_{G}^{*}(M)$, and for this we are going to use a decomposition theorem for orbifold K-theory due to Adem and Ruan [AR03] (cf. [LO01]).

Theorem 2.7 ([AR03, Th. 5.1]). Let $[M / G]$ be a compact orbifold where $G$ is a compact Lie group acting almost freely on $M$. Then there is a rational isomorphism of rings

$$
K_{G}^{*}(M) \otimes \mathbb{Q} \cong \prod_{\{(C) \mid C \subset G \text { cyclic }\}}\left[K^{*}\left(M^{C} / Z_{G}(C)\right) \otimes \mathbb{Q}\left(\zeta_{|C|}\right)\right]^{W_{G}(C)},
$$

where $(C)$ ranges over conjugacy classes of cyclic subgroups, $W_{G}(C)=N_{G}(C) / Z_{G}(C)$ and $\mathbb{Q}\left(\xi_{|C|}\right)$ is the cyclotomic field where $\zeta_{|C|}$ is a primitive $|C|$-th root of unity.

For a cyclic group $C$, the map from left to right is the composition of maps

$$
\begin{aligned}
K_{G}^{*}(M) \stackrel{\phi}{\longrightarrow} & K_{N_{G}(C)}^{*}\left(M^{C}\right) \stackrel{\psi}{\longrightarrow} K_{Z_{G}(C)}^{*}\left(M^{C}\right) \otimes R(C) \\
& \stackrel{\alpha}{\longrightarrow} K^{*}\left(E G \times{ }_{Z_{G}(C)} M^{C}\right) \otimes R(C) \stackrel{\beta}{\longrightarrow} K^{*}\left(M^{C} / Z_{G}(C)\right) \otimes \mathbb{Q}\left(\zeta_{|C|}\right),
\end{aligned}
$$

where: $\phi$ is the restriction map; $\psi$ is defined such that for any $N_{G}(C)$ vector bundle $E \rightarrow M^{C}$

$$
\psi([E])=\sum_{V \in \operatorname{Irr}(C)}\left[\operatorname{Hom}_{C}(V, E)\right] \otimes[V]
$$

$\alpha$ is the natural map from equivariant K-theory to the K-theory of the Borel construction; and $\beta$ is the map obtained from the isomorphism

$$
K^{*}\left(E G \times Z_{G}(C) M^{C}\right) \otimes \mathbb{Q} \cong K^{*}\left(M^{C} / Z_{G(C)}\right) \otimes \mathbb{Q}
$$

that comes from the rational acyclicity of the fibers of the map $E G \times{ }_{Z_{G}(C)} M^{C} \rightarrow$ $M^{C} / Z_{G(C)}$, and the ring map $R(C) \otimes \mathbb{Q} \rightarrow \mathbb{Q}\left(\zeta_{|C|}\right)$ whose kernel is the ideal of elements whose characters vanish in all generators of $C$.

Remark 2.8. We have modified slightly the statement of Theorem 5.1 in AR03 by leaving the decomposition formula only in terms of K-theory. Applying the Chern character isomorphism

$$
C h: K^{*}\left(M^{C} / Z_{G(C)}\right) \otimes \mathbb{Q} \stackrel{\cong}{\longrightarrow} H^{*}\left(M^{C} / Z_{G(C)}\right) \otimes \mathbb{Q},
$$

one recovers the original statement. 
Going back to the weighted projective orbifold $M / G$, we have

Lemma 2.9. Let $G=S^{1}$ act on $M=S^{2 n+1}$ as in (2.3); then there is an isomorphism of rings

where $\zeta=e^{\frac{2 \pi i}{p}}$.

$$
K_{G}^{*}(M) \otimes \mathbb{Q} \cong \mathbb{Q}[u] /\left\langle(1-u)^{n+1}\right\rangle \times \mathbb{Q}(\zeta)
$$

Proof. From Theorem 2.7 we have that

$$
K_{G}^{*}(M) \otimes \mathbb{Q} \cong\left(K^{*}(M / G) \otimes \mathbb{Q}\right) \times\left(K^{*}\left(M^{\mathbb{Z} / p} / G\right) \otimes \mathbb{Q}(\zeta)\right),
$$

because the only cyclic subgroups of the circle that have a non-empty fixed point set are the trivial subgroup and $\mathbb{Z} / p$. The quotient spaces are

$$
M / G=\mathbb{C} P(p: 1: \cdots: 1)
$$

and $M^{\mathbb{Z} / p} / G=$ point; therefore

$$
K_{G}^{*}(M) \otimes \mathbb{Q} \cong\left(K^{*}(\mathbb{C} P(p: 1: \cdots: 1)) \otimes \mathbb{Q}\right) \times \mathbb{Q}(\zeta) .
$$

We are left to calculate the $\mathrm{K}$-theory of the weighted projective space $\mathbb{C} P_{w}^{n}:=$ $\mathbb{C} P(p: 1: \cdots: 1)$; this we will do by calculating its cohomology.

The space $\mathbb{C} P_{w}^{n}$ has only one orbifold point whose neighborhood looks like the quotient of $\mathbb{C}^{n}$ by the diagonal action of $\mathbb{Z} / p$. Then the space $\mathbb{C} P_{w}^{n}$ can be thought as a smooth manifold because the orbifold point can be removed.

If we consider the inclusion $j: \mathbb{C} P^{n-1} \rightarrow \mathbb{C} P_{w}^{n}$ that skips the first variable, then we get the following short exact sequence:

$$
0 \longrightarrow H^{2 n}\left(\mathbb{C} P_{w}^{n}, \mathbb{C} P^{n-1}\right) \longrightarrow H^{*}\left(\mathbb{C} P_{w}^{n}\right) \stackrel{j^{*}}{\longrightarrow} H^{*}\left(\mathbb{C} P^{n-1}\right) \longrightarrow 0 .
$$

As $H^{*}\left(\mathbb{C} P^{n-1}\right)=\mathbb{Z}[y] /\left\langle y^{n}\right\rangle$, there must exist $x \in H^{2}\left(\mathbb{C} P_{w}^{n}\right)$ with $j^{*} x=y$. As the transversal intersection of the submanifold $j\left(\mathbb{C} P^{n-1}\right)$ with itself $n-1$ times inside $\mathbb{C} P_{w}^{n}$ gives $p$ points (this is because the normal bundle of the inclusion $j$ is isomorphic to the $p$-th power of the canonical line bundle over $\left.\mathbb{C} P^{n-1}\right)$, then $x^{n}$ must be $p$-times the image of the generator of $H^{2 n}\left(\mathbb{C} P_{w}^{n}, \mathbb{C} P^{n-1}\right)$.

Therefore we can conclude that

$$
H^{*}\left(\mathbb{C} P_{w}^{n}\right) \cong \mathbb{Z}[x, v] /\left\langle x^{n}-p v, x^{n+1}, v^{2}\right\rangle,
$$

with rational coefficients and taking $z:=p^{-\frac{1}{n}} x$

$$
H^{*}\left(\mathbb{C} P_{w}^{n}, \mathbb{Q}\right) \cong \mathbb{Q}[z] /\left\langle z^{n+1}\right\rangle,
$$

and by the Chern character isomorphism

$$
K^{*}\left(\mathbb{C} P_{w}^{n}\right) \otimes \mathbb{Q} \cong \mathbb{Q}[u] /\left\langle(1-u)^{n+1}\right\rangle,
$$

where $\operatorname{ch}(1-u)^{n}=z^{n}$.

For the fixed point set $M^{\mathbb{Z} / p}$ we already know that $K_{G}\left(M^{\mathbb{Z} / p}\right) \cong R(\mathbb{Z} / p) \cong$ $\mathbb{Z}[W] /\left\langle W^{p}-1\right\rangle$. Applying Theorem 2.7 again we have that

Lemma 2.10. There is a ring isomorphism

$$
\begin{aligned}
R(\mathbb{Z} / p) \otimes \mathbb{Q} & \cong \mathbb{Q} \otimes \mathbb{Q}(\zeta), \\
f(W) & \mapsto(f(1), f(\zeta)),
\end{aligned}
$$

where $f(W)$ is a polynomial that belongs to $\mathbb{Z}[W] /\left\langle W^{p}-1\right\rangle$ and $W$ is the one dimensional irreducible representation of $\mathbb{Z} / p$ whose character on $1 \in \mathbb{Z} / p$ is $e^{\frac{2 \pi i}{p}}$. 
Lemma 2.11. The pullback of the inclusion map $i: M^{\mathbb{Z} / p} \rightarrow M$,

$$
i^{*}: K_{G}^{*}(M) \otimes \mathbb{Q} \rightarrow K_{G}^{*}\left(M^{\mathbb{Z} / p}\right) \otimes \mathbb{Q}
$$

is isomorphic to

$$
\begin{aligned}
\mathbb{Q}[u] /\left\langle(1-u)^{n+1}\right\rangle \times \mathbb{Q}(\zeta) & \rightarrow \mathbb{Q} \times \mathbb{Q}(\zeta), \\
(p(u), q(\zeta)) & \mapsto(p(1), q(\zeta)) .
\end{aligned}
$$

Proof. It follows directly from Lemmas 2.10 and 2.9

The pushforward $i_{\text {! }}$ of the inclusion follows the same lines.

Lemma 2.12. The pushforward

$$
i_{!}: K_{G}^{*}\left(M^{\mathbb{Z} / p}\right) \otimes \mathbb{Q} \rightarrow K_{G}^{*}(M) \otimes \mathbb{Q}
$$

is isomorphic to

$$
\begin{aligned}
i_{!}: \mathbb{Q} \times \mathbb{Q}(\zeta) & \rightarrow \mathbb{Q}[u] /\left\langle(1-u)^{n+1}\right\rangle \times \mathbb{Q}(\zeta), \\
(r, q(\zeta)) & \mapsto\left(r(1-u)^{n}, q(\zeta)(1-\zeta)^{n}\right) .
\end{aligned}
$$

Proof. The application in the first coordinate is the pushforward in K-theory of the inclusion of a point into the weighted projective space $\mathbb{C} P_{w}^{n}$, and the application of the second coordinate is the image in $\mathbb{Q}(\zeta)$ of the map $i^{*} i_{!}: R(\mathbb{Z} / p) \rightarrow R(\mathbb{Z} / p)$

$$
i^{*} i_{!} q(W)=q(W) \lambda_{-1}(n W)=q(W) \lambda_{-1}(W)^{n}=q(\xi)(1-W)^{n},
$$

where $n W$ represents the class of the normal bundle of the inclusion $i$ as a $G$ equivariant bundle.

We can finish this section by giving the explicit calculation of the stringy product in the orbifold K-theory of $[M / G]=\mathbb{C} P[p: 1: \cdots: 1]$.

Theorem 2.13. The stringy ring with rational coefficients of the K-theory of the inertia orbifold of the weighted projective orbifold $[M / G]=\mathbb{C} P[p: 1: \cdots: 1]$ is

$$
K_{s t}^{*}([M / G]) \otimes \mathbb{Q} \cong\left(\mathbb{Q}[u] /\left\langle(u-1)^{n+1}\right\rangle \times \mathbb{Q}(\zeta)\right) \times\left\{g_{0}\right\} \oplus \bigoplus_{j=1}^{p-1}(\mathbb{Q} \times \mathbb{Q}(\zeta)) \times\left\{g_{j}\right\},
$$

with the product defined by the following cases:

- $(j=0, k \neq 0)$

$$
\left(p(u), q(\zeta), g_{0}\right) \star\left(r, w(\zeta), g_{k}\right)=\left(p(1) r, q(\zeta) w(\zeta), g_{k}\right) .
$$

- $(j+k<p, j \neq 0, k \neq 0)$

$$
\left(r, q(\zeta), g_{j}\right) \star\left(s, w(\zeta), g_{k}\right)=\left(r s, q(\zeta) w(\zeta), g_{j+k}\right) .
$$

- $(j+k=p, j \neq 0, k \neq 0)$

$$
\left(r, q(\zeta), g_{j}\right) \star\left(s, w(\zeta), g_{k}\right)=\left(r s(1-u)^{n}, q(\zeta) w(\zeta)(1-\zeta)^{n}, g_{0}\right) .
$$

- $(j+k>p)$

$$
\left(r, q(\zeta), g_{j}\right) \star\left(s, w(\zeta), g_{k}\right)=\left(0, q(\zeta) w(\zeta)(1-\zeta)^{n}, g_{j+k-p}\right) .
$$

Proof. From formula (2.4) we know that if the obstruction bundle is different than zero, then it is precisely the normal bundle. Therefore the Euler class of the obstruction bundle is 1 in the case that the bundle is zero, and $(1-\zeta)^{n}$ in the case that it is the normal bundle. The product structure $\star$ follows from Definition 2.5 and Lemmas 2.9 and 2.10 . 
2.5. Relation with Chen-Ruan cohomology. In what follows we want to prove that there is a homomorphism of rings from the stringy ring of the K-theory of the inertia orbifold to the Chen-Ruan cohomology. We will show this fact by first constructing a ring structure on the K-theory of the Borel construction of the inertia orbifold. Then, via a modified version of the Chern character map, we will construct a ring structure on the cohomology with rational coefficients of the Borel construction of the inertia orbifold. This latter ring, once tensored with $\mathbb{Q}$, will be isomorphic to the Chen-Ruan cohomology.

2.6. Stringy K-theory for the Borel construction of the inertia orbifold. Let's start by reviewing the relation between equivariant K-theory and the K-theory of the Borel construction (see AS69).

For any $G$-space $N$ with $N_{G}:=N \times_{G} E G$, let's denote the map $\tau_{N}: K_{G}(N) \rightarrow$ $K\left(N_{G}\right)$ that takes any $G$-equivariant complex vector bundle $F$ over $N$ and maps it to $\tau_{N}(F)=F_{G}:=F \times_{G} E G$, a complex vector bundle over $N_{G}$. This map is a ring homomorphism and has very nice properties: first, it behaves well under restriction and pushforwards; namely, if we have an inclusion of $G$-manifolds $i: W \rightarrow N$, then

$$
\tau_{W} i^{*}(L)=i^{*} \tau_{N}(L) \quad \text { and } \quad \tau_{N} i_{!}(F)=i_{!} \tau_{W}(F) ;
$$

and second, it behaves well under the map $\lambda_{-1}$, i.e.

$$
\tau_{N}\left(\lambda_{-1}(L)\right)=\lambda_{-1}\left(\tau_{N}(L)\right)
$$

Moreover, the map $\tau_{N}$ realizes the famous Atiyah-Segal completion theorem [AS69.

Theorem 2.14. Let the orbifold $[M / G]$ be as in Section 2 . The K-theory of the Borel construction of the inertia orbifold

$$
K_{o r b}^{*}([M / G]):=\bigoplus_{g \in \mathcal{C}} K^{*}\left(M_{G}^{g}\right) \times\{g\}
$$

can be endowed with the ring structure

$$
\text { •: } \begin{aligned}
\left(K^{*}\left(M_{G}^{g}\right) \times\{g\}\right) \times\left(K^{*}\left(M_{G}^{h}\right) \times\{h\}\right) & \rightarrow K^{*}\left(M_{G}^{g h}\right) \times\{g h\}, \\
((F, g),(H, h)) & \mapsto(F \bullet H, g h),
\end{aligned}
$$

with $F \bullet H=e_{3 !}\left(e_{1}^{*} F \otimes e_{2}^{*} H \otimes \lambda_{-1}\left(\tau_{M^{g, h}}\left(D_{g, h}\right)\right)\right.$.

Proof. The associativity of the product $\bullet$ follows from the associativity of $\star$ (see Section 2.3) and the properties of the maps $\tau$.

Remark 2.15. The construction above of Theorem 2.14 is due to Jarvis-KaufmannKimura [JKK07] when one considers the coarse moduli space of the inertia orbifold. Ours generalizes it to the Borel construction.

Theorem 2.16. The maps

$$
\tau_{M^{g}}: K_{G}^{*}\left(M^{g}\right) \rightarrow K^{*}\left(M_{G}^{g}\right)
$$

for $g \in \mathcal{C}$ define a ring homomorphism

$$
\tau:\left(K_{s t}^{*}([M / G]), \star\right) \rightarrow\left(K_{\text {orb }}^{*}([M / G]), \bullet\right) .
$$


Proof. Take $F \in K_{G}^{*}\left(M^{g}\right)$ and $H \in K_{G}^{*}\left(M^{h}\right)$ and consider the following set of equalities:

$$
\begin{aligned}
\tau_{M^{g h}}(F \star H) & =\tau_{M^{g h}}\left(e_{3 !}\left(e_{1}^{*} F \otimes e_{2}^{*} H \otimes \lambda_{-1}\left(D_{g, h}\right)\right)\right) \\
& =e_{3 !}\left(\tau_{M^{g, h}}\left(e_{1}^{*} F \otimes e_{2}^{*} H \otimes \lambda_{-1}\left(D_{g, h}\right)\right)\right) \\
& =e_{3 !}\left(e_{1}^{*}\left(\tau_{M^{g}} F\right) \otimes e_{2}^{*}\left(\tau_{M^{h}} H\right) \otimes \lambda_{-1}\left(\tau_{M^{g, h}} D_{g, h}\right)\right) \\
& =\tau_{M^{g}} F \bullet \tau_{M^{h}} H .
\end{aligned}
$$

The theorem follows.

2.7. Chern character. We can define a stringy product on the cohomology of the Borel construction of the inertia orbifold of $[M / G]$ in the same way that it was done in the previous section.

Proposition 2.17. The cohomology of the Borel construction of the inertia orbifold

$$
H_{\text {orb }}^{*}([M / G]):=\bigoplus_{g \in \mathcal{C}} H^{*}\left(M_{G}^{g}, \mathbb{Z}\right) \times\{g\}
$$

can be endowed with the ring structure

$$
\begin{aligned}
\circ:\left(H^{*}\left(M_{G}^{g}, \mathbb{Z}\right) \times\{g\}\right) \times\left(H^{*}\left(M_{G}^{h}, \mathbb{Z}\right) \times\{h\}\right) & \rightarrow H^{*}\left(M_{G}^{g h}, \mathbb{Z}\right) \times\{g h\}, \\
((\alpha, g),(\beta, h)) & \mapsto(\alpha \circ \beta, g h),
\end{aligned}
$$

with $\alpha \circ \beta=e_{3 *}\left(e_{1}^{*} \alpha \cdot e_{2}^{*} \beta \cdot \mathrm{eu}\left(\tau_{M^{g, h}}\left(D_{g, h}\right)\right)\right.$.

Proof. The associativity of the product $\circ$ also follows from the associativity of product $\star$ (see Section 2.3) , the properties of the map $\tau_{M^{g, h}}$ and the properties of the Euler class "eu".

In order to have a well defined ring homomorphism between $\left(K_{\text {orb }}^{*}([M / G]), \bullet\right)$ and $\left(H_{o r b}^{*}([M / G]), \circ\right)$, we need to calibrate the Chern character maps at the level of the spaces $M_{G}^{g}$ with some invertible elements in cohomology. This construction was developed in [JKK07] at the level of the coarse moduli space of the inertia orbifold. The map is basically the same at the level of the Borel construction.

To construct this calibrated Chern character we need to recall some properties of the Thom ismorphisms in K-theory and in cohomology (see for instance AS68, section 2]).

Let $X$ be a manifold and $V$ a complex vector bundle over $X$. Let

$$
\phi: K^{*}(X) \rightarrow K^{*}(V), \psi: H^{*}(X, \mathbb{Q}) \rightarrow H^{*}(V, \mathbb{Q})
$$

be the Thom isomorphisms in K-theory and in cohomology respectively. Then, for any $u \in K^{*}(X)$ one has

$$
\operatorname{ch}(\phi(u))=\psi(\operatorname{ch}(u) \cdot \mu(V)),
$$

where the cohomology class $\mu(V)$ is defined as

$$
\mu(V):=\prod \frac{1-e^{x_{i}}}{x_{i}}
$$

where the $x_{i}$ 's are the Chern roots of $V$. Moreover, the class $\mu(V)$ is multiplicative, i.e. $\mu(V \oplus F)=\mu(V) \cdot \mu(F)$, and measures the difference of the Chern character of the Euler class in K-theory with the one in cohomology, namely

$$
\operatorname{ch} \lambda_{-1}(V)=\mathrm{eu}(V) \cdot \mu(V) .
$$


If we have an inclusion of manifolds $i: X \rightarrow Y$ with normal bundle $V$ and pushforward maps

$$
\begin{gathered}
i_{!}: K^{*}(X) \rightarrow K^{*}(Y), \\
i_{*}: H^{*}(X, \mathbb{Q}) \rightarrow H^{*}(Y, \mathbb{Q}),
\end{gathered}
$$

then one has the equality

$$
\operatorname{ch}\left(i_{!} u\right)=i_{*}(\operatorname{ch}(u) \cdot \mu(V)),
$$

where in this case the cohomology class $\mu(V)$ has support on the normal bundle of $X$.

We are now ready to define the Chern character map for the orbifold K-theory.

Definition 2.18 ([JKK07, Formula (55)]). The orbifold Chern character is the homomorphism

$$
C h: K_{\text {orb }}^{*}([M / G]) \rightarrow H_{o r b}^{*}([M / G] ; \mathbb{Q}),
$$

such that for all $g \in \mathcal{C}$ is defined as

$$
\begin{aligned}
C h: K^{*}\left(M_{G}^{g}\right) & \rightarrow H^{*}\left(M_{G}^{g} ; \mathbb{Q}\right), \\
F & \mapsto \operatorname{ch}(F) \mu^{-1}\left(\tau_{M^{g}} \mathcal{F}_{g}\right),
\end{aligned}
$$

where the $G$-bundle $\mathcal{F}_{g}$ over $M^{g}$ is defined in formula (2.1).

The fact that the orbifold Chern character is a ring homomorphism depends on the following lemma.

Lemma 2.19. In $K_{G}\left(M^{g, h}\right)$ the following equality holds:

$$
\left.D_{g, h} \oplus T M^{g h}\right|_{M^{g, h}} \ominus T M^{g, h}=\ominus e_{1}^{*} \mathcal{F}_{g} \ominus e_{2}^{*} \mathcal{F}_{h} \oplus e_{3}^{*} \mathcal{F}_{g h} .
$$

Proof. We know that

$$
\mathcal{F}_{g h} \oplus \mathcal{F}_{(g h)^{-1}}=\left.T M\right|_{M^{g h}} \ominus T M^{g h} .
$$

The equality then holds from the fact that $D_{g, h}=B_{g, h}$, where $B_{g, h}$ is defined in formula (2.2).

Applying the homomorphism $\tau_{M^{g, h}}$ and the map $\mu$ we get

\section{Corollary 2.20.}

$$
\begin{aligned}
& \mu\left(\tau_{M^{g, h}} D_{g, h}\right) \mu\left(\tau_{M^{g, h}}\left(\left.T M^{g h}\right|_{M^{g, h}} \ominus T M^{g, h}\right)\right) e_{3}^{*} \mu^{-1}\left(\tau_{M^{g h}} \mathcal{F}_{g h}\right) \\
& \quad=e_{1}^{*} \mu^{-1}\left(\tau_{M^{g}} \mathcal{F}_{g}\right) e_{2}^{*} \mu^{-1}\left(\tau_{M^{h}} \mathcal{F}_{h}\right) .
\end{aligned}
$$

Theorem 2.21 ([JKK07, Theorem 6.1]). The orbifold Chern character is a ring homomorphism

$$
C h:\left(K_{\text {orb }}^{*}([M / G]), \bullet\right) \rightarrow\left(H_{\text {orb }}^{*}([M / G] ; \mathbb{Q}), \circ\right) .
$$


Proof. Take $F \in K^{*}\left(M_{G}^{g}\right)$ and $H \in H^{*}\left(M_{G}^{h}\right)$ and consider the following set of equalities:

$$
\begin{aligned}
& C h(F \bullet H)= \operatorname{ch}(F \bullet H) \mu^{-1}\left(\tau_{M^{g h}} \mathcal{F}_{g h}\right) \\
&= \operatorname{ch}\left(e_{3 !}\left(e_{1}^{*} F \otimes e_{2}^{*} H \otimes \lambda_{-1}\left(\tau_{M^{g, h}} D_{g, h}\right)\right) \mu^{-1}\left(\tau_{M^{g h}} \mathcal{F}_{g h}\right)\right. \\
&= e_{3 *}\left(e_{1}^{*} \operatorname{ch} F e_{2}^{*} \operatorname{ch} H \operatorname{eu}\left(\tau_{M^{g, h}} D_{g, h}\right) \mu\left(\tau_{M^{g, h}} D_{g, h}\right)\right. \\
&\left.\quad \mu\left(\tau_{M^{g, h}}\left(\left.T M^{g h}\right|_{M^{g, h}} \ominus T M^{g, h}\right)\right)\right) \mu^{-1}\left(\tau_{M^{g h}} \mathcal{F}_{g h}\right) \\
&= e_{3 *}\left(e_{1}^{*} \operatorname{ch} F e_{2}^{*} \operatorname{ch} H \operatorname{eu}\left(\tau_{M^{g, h}} D_{g, h}\right) \mu\left(\tau_{M^{g, h}} D_{g, h}\right)\right. \\
&\left.\quad \cdot \mu\left(\tau_{M^{g, h}}\left(\left.T M^{g h}\right|_{M^{g, h}} \ominus T M^{g, h}\right)\right) e_{3}^{*} \mu^{-1}\left(\tau_{M^{g h}} \mathcal{F}_{g h}\right)\right) \\
&= e_{3 *}\left(e_{1}^{*} \operatorname{ch} F e_{2}^{*} \operatorname{ch} H \operatorname{eu}\left(\tau_{M^{g, h}} D_{g, h}\right) e_{1}^{*} \mu^{-1}\left(\tau_{M^{g}} \mathcal{F}_{g}\right) e_{2}^{*} \mu^{-1}\left(\tau_{M^{h}} \mathcal{F}_{h}\right)\right) \\
&= e_{3 *}\left(e_{1}^{*} C h F e_{2}^{*} C h H \operatorname{eu}\left(\tau_{M^{g, h}} D_{g, h}\right)\right) \\
&= C h F \circ C h H .
\end{aligned}
$$

From the second to the third line we used formula (2.6), the fourth line is by the properties of the pushforward and the fifth line is by Corollary 2.20 .

Using the fact that the Chern character map becomes a $\mathbb{Z} / 2$ graded vector space isomorphism when one tensors the K-theory with the rational numbers, and because the classes given by $\mu$ are all invertible, we can deduce that

Corollary 2.22. The orbifold Chern character is a ring isomorphism when one tensors the orbifold K-theory with the rationals, i.e.

$$
C h:\left(K_{o r b}^{*}([M / G]) \otimes \mathbb{Q}, \bullet\right) \stackrel{\cong}{\rightrightarrows}\left(H_{o r b}^{*}([M / G] ; \mathbb{Q}), \circ\right)
$$

is a ring isomorphism.

2.8. Chen-Ruan cohomology. The Chen-Ruan cohomology is defined at the level of the quotient spaces $M^{g} / G$, where the obstruction class is the Euler class of the orbibundle $D_{g, h} / G$ over $M^{g, h} / G$ (see [CR04]). Namely

$$
H_{C R}^{*}([M / G]):=\bigoplus_{g \in \mathcal{C}} H^{*}\left(M^{g} / G, \mathbb{Q}\right)
$$

with the product structure given by

$$
\alpha \cdot \beta:=e_{3 *}\left(e_{1}^{*} \alpha e_{2}^{*} \beta \mathrm{eu}\left(D_{g, h} / G\right)\right) .
$$

As the projection maps $\pi_{g}: M_{G}^{g} \rightarrow M^{g} / G$ induce isomorphisms in cohomology

$$
\pi_{g}^{*}: H^{*}\left(M^{g} / G, \mathbb{Q}\right) \stackrel{\cong}{\rightrightarrows} H^{*}\left(M_{G}^{g} ; \mathbb{Q}\right)
$$

(because the cohomology with rational coefficients of the fibers of $\pi_{g}$ are acyclic) and commute with pullbacks and pushforwards, we can conclude

Theorem 2.23. The maps $\pi_{g}$ induce an isomorphism of rings

$$
\pi^{*}: H_{C R}^{*}([M / G]) \rightarrow H_{\text {orb }}^{*}([M / G], \mathbb{Q}) .
$$

Proof. The isomorphism follows from the fact that

$$
\pi_{g, h}^{*}\left(D_{g, h} / G\right) \cong \tau_{M^{g, h}} D_{g, h} .
$$


Composing the ring maps $\tau, C h$ and $\pi^{*}$ we obtain

Corollary 2.24. The composition

$$
\pi^{*} \circ C h \circ \tau: K_{s t}^{*}([M / G]) \rightarrow H_{C R}^{*}([M / G])
$$

is a ring homomorphism.

Example 2.25. The case of $\mathbb{C} P[p: 1: \cdots: 1]$.

In the case of the weighted projective orbifold that we elaborated in Section 2.4, we just need to disregard the coordinate of the cyclotomic extension of Theorem 2.13: then we have that

$$
K_{\text {orb }}^{*}([M / G]) \otimes \mathbb{Q} \cong \mathbb{Q}[u, g] /\left\langle(1-u)^{n+1}, g^{p}-(1-u)^{n}, g^{p+1}\right\rangle .
$$

Applying the orbifold Chern character, which in this case is simply the Chern character map, we obtain the Chen-Ruan orbifold cohomology of the weighted projective space:

$$
H_{\text {orb }}^{*}(\mathbb{C} P[p: 1: \cdots: 1]) \cong \mathbb{Q}[x, g] /\left\langle x^{n+1}, g^{p}-x^{n}, g^{p+1}\right\rangle,
$$

where $\operatorname{ch}(1-u)^{n}=x^{n}$.

\section{Stringy PROduCt ON TWISTED ORBIFOLD K-TheORY}

This chapter is devoted to the study of some properties of the stringy product on twisted orbifold K-theory for orbifolds of the type $[M / G]$ where $G$ is a abelian finite group.

We will first recall the definition of the twisted orbifold K-theory and its stringy product following [ARZ]. Then we will show that the twistings used for the stringy product that come from the inverse transgression are all torsion. In the case that the twistings come from the cohomology of the group $G$, we will show a decomposition formula that is very suited to the study of the stringy product. This decomposition formula can be seen as a hybrid between the ring decomposition of equivariant K-theory (see AR03. Thm 5.1]) and the group decomposition of twisted K-theory (see [AR03, Thm. 7.4]). Finally we will show an explicit calculation of the stringy product of twisted orbifold K-theory on which the twistings are non-trivial.

3.1. Stringy product on twisted orbifold K-theory. In this section we will review the definition of the twisted orbifold K-theory. For the details on the construction see AR03, ARZ.

An element $\beta \in H_{G}^{3}(M ; \mathbb{Z})$ defines ${ }^{\beta} K_{G}^{*}(M)$, the twisted $G$-equivariant K-theory of $M$. If $\beta$ comes from $H^{3}(B G ; \mathbb{Z})$, then $\beta$ defines a central extension of $G$ by the circle

$$
1 \rightarrow S^{1} \rightarrow \widetilde{G} \rightarrow G \rightarrow 0,
$$

and the twisted $G$-equivariant K-theory ${ }^{\beta} K_{G}^{*}(M)$ becomes the group of $\widetilde{G}$ equivariant vector bundles over $M$ such that $S^{1}$ acts by multiplication on the fibers.

For $\alpha \in H_{G}^{4}(M ; \mathbb{Z})$ the stringy product of the twisted orbifold K-theory ${ }^{\alpha} K_{s t}^{*}([M / G])$ is defined as the group

$$
{ }^{\alpha} K_{s t}^{*}([M / G]):=\bigoplus_{g \in G}^{\alpha_{g}} K_{G}^{*}\left(M^{g}\right) \times\{g\},
$$

where $\alpha_{g} \in H_{G}^{3}\left(M^{g} ; \mathbb{Z}\right)$ is a twisting class that is the image of $\alpha$ under the inverse transgression map $\tau_{g}: H_{G}^{4}(M ; \mathbb{Z}) \rightarrow H_{G}^{3}\left(M^{g} ; \mathbb{Z}\right)($ see $(3.1)$ ). 
Let's denote the inclusions by

$$
e_{1}: M^{g, h} \rightarrow M^{g} \quad e_{2}: M^{g, h} \rightarrow M^{h} \quad e_{3}: M^{g, h} \rightarrow M^{g h},
$$

and recall that $D_{g, h} \in K_{G}^{*}\left(M^{g, h}\right)$ is the obstruction bundle of Definition 2.2.

Definition 3.1. For the almost complex orbifold $[M / G]$, define the stringy product $\star$ in the twisted orbifold K-theory ${ }^{\alpha} K_{s t}^{*}([M / G])$ as follows:

$$
\begin{aligned}
\left({ }^{\alpha_{g}} K_{G}^{*}\left(M^{g}\right) \times\{g\}\right) \times\left({ }^{\alpha_{h}} K_{G}^{*}\left(M^{h}\right) \times\{h\}\right) & \stackrel{\star}{\rightarrow}{ }^{\alpha_{g h}} K_{G}^{*}\left(M^{g h}\right) \times\{g h\}, \\
((F, g),(H, h)) & \mapsto\left(e_{3 !}\left(e_{1}^{*} F \otimes e_{2}^{*} H \otimes \lambda_{-1}\left(D_{g, h}\right)\right), g h\right) .
\end{aligned}
$$

The proof of the fact that the $\star$ operation makes ${ }^{\alpha} K_{s t}^{*}([M / G])$ into a ring can be found in ARZ. The product is well defined because $\alpha_{g}+\alpha_{h}=\alpha_{g h}$, and the associativity of $\star$ follows from the properties of the obstruction bundles as in Section 2.1 .

3.2. Inverse transgression map. In this section we will show that the transgressed classes $\alpha_{g}$ are all torsion.

Let us recall the definition of the inverse transgression map for the orbifold $[M / G]$ when $G$ is finite not necessarily abelian.

For $g \in G$ let $C(g)$ denote the centralizer of $g$ in $G$. Consider the action of $C(g) \times \mathbb{Z}$ on $M^{g}$ given by $(h, m) \cdot x:=x h g^{m}$ and the homomorphism

$$
\begin{aligned}
\phi_{g}: C(g) \times \mathbb{Z} & \rightarrow G, \\
(h, m) & \mapsto h g^{m} .
\end{aligned}
$$

Then the inclusion $i_{g}: M^{g} \rightarrow M$ becomes a $\phi_{g}$-equivariant map and it induces a homomorphism

$$
i_{g}^{*}: H_{G}^{*}(M ; \mathbb{Z}) \rightarrow H_{C(g) \times Z}^{*}\left(M^{g} ; \mathbb{Z}\right) .
$$

As the group $\mathbb{Z}$ acts trivially on $M^{g}$, we have that

$$
H_{C(g) \times Z}^{*}\left(M^{g} ; \mathbb{Z}\right) \cong H^{*}\left(M^{g} \times_{C(g)} E C(g) \times B \mathbb{Z} ; \mathbb{Z}\right),
$$

and as $B \mathbb{Z} \simeq S^{1}$, via the Kunneth isomorphism we get that

$$
H_{C(g) \times Z}^{*}\left(M^{g} ; \mathbb{Z}\right) \cong H_{C(g)}^{*}\left(M^{g} ; \mathbb{Z}\right) \otimes_{\mathbb{Z}} H^{*}\left(S^{1} ; \mathbb{Z}\right) .
$$

Therefore

$$
i_{g}^{*}: H_{G}^{p}(M ; \mathbb{Z}) \rightarrow H_{C(g)}^{p}\left(M^{g} ; \mathbb{Z}\right) \oplus H_{C(g)}^{p-1}\left(M^{g} ; \mathbb{Z}\right),
$$

and projecting on the second summand we get the inverse transgression map:

$$
\tau_{g}: H_{G}^{p}(M ; \mathbb{Z}) \rightarrow H_{C(g)}^{p-1}\left(M^{g} ; \mathbb{Z}\right) .
$$

Lemma 3.2. The transgressed classes are all torsion, i.e. for $\alpha \in H_{G}^{p}(M ; \mathbb{Z})$ the inverse transgression $\alpha_{g}:=\tau_{g}(\alpha) \in H_{C(g)}^{p-1}\left(M^{g} ; \mathbb{Z}\right)$ is a torsion class.

Proof. Let $\langle g\rangle$ denote the cyclic group generated by $g$. Then the homomorphism $\phi_{g}$ factors through

$$
C(g) \times \mathbb{Z} \rightarrow C(g) \times\langle g\rangle \rightarrow G \quad(h, m) \mapsto\left(h, g^{m}\right) \mapsto h g^{m},
$$

and therefore $i_{g}^{*}$ factors through

$$
H_{G}^{*}(M ; \mathbb{Z}) \rightarrow H^{*}\left(M^{g} \times_{C(g)} E C(g) \times B\langle g\rangle ; \mathbb{Z}\right) \rightarrow H^{*}\left(M^{g} \times_{C(g)} E C(g) \times B \mathbb{Z} ; \mathbb{Z}\right) .
$$


Now, as the cohomology of $B\langle g\rangle$ is all torsion, the Kunneth isomorphism tells us that the torsion free part of $H^{p}\left(M^{g} \times_{C(g)} E C(g) \times B\langle g\rangle ; \mathbb{Z}\right)$ comes from

$$
H_{C(g)}^{p}\left(M^{g} ; \mathbb{Z}\right) \otimes_{\mathbb{Z}} H^{0}(B\langle g\rangle ; \mathbb{Z}) .
$$

As the inverse transgression map does not factor through $H_{C(g)}^{p}\left(M^{g} ; \mathbb{Z}\right)$ $\otimes_{\mathbb{Z}} H^{0}(B\langle g\rangle ; \mathbb{Z})$, then the transgressed classes must be torsion.

Example 3.3. Let's calculate the inverse transgression map for the group $G=$ $(\mathbb{Z} / 2)^{3}$; here we follow the analysis of lemma 5.2 in [ARZ.

Let's suppose first that the coefficients are in the field $\mathbb{F}_{2}$ of two elements. Then we have that

$$
H^{*}\left(G ; \mathbb{F}_{2}\right) \cong \mathbb{F}_{2}\left[x_{1}, x_{2}, x_{3}\right]
$$

is the polynomial algebra on three generators. Take $g=\left(a_{1}, a_{2}, a_{3}\right) \in G$ and consider the maps

$$
G \times \mathbb{Z} \rightarrow G \times \mathbb{Z} / 2 \rightarrow G \quad(h, m) \mapsto(h, m g) \mapsto h+m g .
$$

In cohomology we get

$$
\begin{aligned}
& H^{*}\left(B G, \mathbb{F}_{2}\right) \rightarrow H^{*}\left(B G \times B \mathbb{Z} / 2 ; \mathbb{F}_{2}\right) \rightarrow H^{*}\left(B G \times B \mathbb{Z} ; \mathbb{F}_{2}\right), \\
& \mathbb{F}_{2}\left[x_{1}, x_{2}, x_{3}\right] \rightarrow \mathbb{F}_{2}\left[x_{1}, x_{2}, x_{3}, w\right] \rightarrow \mathbb{F}_{2}\left[x_{1}, x_{2}, x_{3}, w\right] /\left(w^{2}\right), \\
& x_{i} \mapsto x_{i}+a_{i} w \quad \mapsto x_{i}+a_{i} w,
\end{aligned}
$$

and therefore the inverse transgression map with $\mathbb{F}_{2}$ coefficients is

$$
\begin{gathered}
\tau_{g}: \mathbb{F}_{2}\left[x_{1}, x_{2}, x_{3}\right] \rightarrow \mathbb{F}_{2}\left[x_{1}, x_{2}, x_{3}\right], \\
x_{1}^{m_{1}} x_{2}^{m_{2}} x_{3}^{m_{3}} \mapsto\left(a_{1} m_{1} x_{1}^{m_{1}-1} x_{2}^{m_{2}} x_{3}^{m_{3}}+a_{2} m_{2} x_{1}^{m_{1}} x_{2}^{m_{2}-1} x_{3}^{m_{3}}+a_{3} m_{3} x_{1}^{m_{1}} x_{2}^{m_{2}} x_{3}^{m_{3}-1}\right) .
\end{gathered}
$$

For abelian 2-groups, the map $H^{k}(B G ; \mathbb{Z}) \rightarrow H^{k}\left(B G ; \mathbb{F}_{2}\right)$ is a monomorphism, and as $H^{k}(B G ; \mathbb{Z}) \cong H^{k}(B G ; \mathbb{Z} / 4)$, the long exact sequence induced by the extension $0 \rightarrow \mathbb{Z} / 2 \rightarrow \mathbb{Z} / 4 \rightarrow \mathbb{Z} / 2 \rightarrow 0$ implies that

$$
H^{k}(B G ; \mathbb{Z}) \cong \operatorname{kernel}\left(S q^{1}: H^{k}\left(B G ; \mathbb{F}_{2}\right) \rightarrow H^{k+1}\left(B G ; \mathbb{F}_{2}\right)\right) ;
$$

here $S q^{1}$ is the Steenrod square operation that in this case is equivalent to the Bockstein map.

If we want to find elements in $H^{4}(B G ; \mathbb{Z})$ that induce non-trivial twistings via the inverse transgression map, one needs to find a homogenous polynomial $p\left(x_{1}, x_{2}, x_{3}\right) \in \mathbb{F}_{2}\left[x_{1}, x_{2}, x_{3}\right]$ of degree 4 , such that $S q^{1}(p)=0$ and that $\tau_{g}(p) \neq 0$ for some $g \in G$. In [ARZ, Lemma 5.2] it is shown that the element

$$
\alpha=S q^{1}\left(x_{1} x_{2} x_{3}\right)=x_{1}^{2} x_{2} x_{3}+x_{1} x_{2}^{2} x_{3}+x_{1} x_{2} x_{3}^{2}
$$

satisfies these properties. Its inverse transgression in $\tau_{g}: H^{4}(B G ; \mathbb{Z}) \rightarrow H^{3}(B G, \mathbb{Z})$ for $g=\left(a_{1}, a_{2}, a_{3}\right)$ is equal to

$$
\alpha_{g}=\tau_{g}(\alpha)=a_{1}\left(x_{2}^{2} x_{3}+x_{2} x_{3}^{2}\right)+a_{2}\left(x_{3}^{2} x_{1}+x_{3} x_{1}^{2}\right)+a_{3}\left(x_{1}^{2} x_{2}+x_{1} x_{2}^{2}\right) .
$$

Let us calculate the double inverse transgression of $\alpha$.

Lemma 3.4. Let $g=\left(a_{1}, a_{2}, a_{3}\right)$ and $h=\left(b_{1}, b_{2}, b_{3}\right)$ be elements in $G=(\mathbb{Z} / 2)^{3}$. The double inverse transgression of $\alpha$ is equal to

$$
\begin{aligned}
\tau_{h} \tau_{g} \alpha & =\left(a_{2} b_{3}+a_{3} b_{2}\right) x_{1}^{2}+\left(a_{3} b_{1}+a_{1} b_{3}\right) x_{2}^{2}+\left(a_{1} b_{2}+a_{2} b_{1}\right) x_{3}^{2} \\
& =\left[\left(a_{1}, a_{2}, a_{3}\right) \times\left(b_{1}, b_{2}, b_{3}\right)\right] \cdot\left(x_{1}^{2}, x_{2}^{2}, x_{3}^{2}\right),
\end{aligned}
$$

and therefore $\tau_{h} \tau_{g} \alpha \neq 0$ if and only if $g \neq h$ and $g \neq 0 \neq h$. 
Proof. The formula follows from the fact that the inverse transgression $\tau_{h}\left(x_{i}^{2} x_{j}\right)$ is $b_{j} x_{i}^{2}$. Now, the cross product $\left(a_{1}, a_{2}, a_{3}\right) \times\left(b_{1}, b_{2}, b_{3}\right)$ is equal to zero only when either $g=0$ or $h=0$, or $g=h$.

3.3. Decomposition formula for twisted orbifold K-theory. This section is devoted to showing a decomposition formula for the twisted orbifold K-theory that is suited to the study of the stringy product; this formula is a simple generalization of [AR03, Thm. 7.4]. In what follows we will elaborate on the results of sections 5 and 6 of AR03.

Let's assume that $G$ is abelian and that $\beta: G \times G \rightarrow S^{1}$ is a cocycle representing the twisting class which corresponds to the group extension $1 \rightarrow S^{1} \rightarrow \widetilde{G}_{\beta} \rightarrow G \rightarrow$ 0 . Therefore $\widetilde{G}_{\beta}$ is isomorphic to the group $G \times S^{1}$ with the product given by $\left(g_{1}, c_{1}\right)\left(g_{2}, c_{2}\right)=\left(g_{1} g_{2}, \beta\left(g_{1}, g_{2}\right) c_{1} c_{2}\right)$.

We will start by recalling some fact about projective representations that can be found in AR03. Recall that the group $R_{\beta}(G):={ }^{\beta} K_{G}^{*}(p t)$ of $\beta$-twisted representations can be understood as the subgroup of $R\left(\widetilde{G}_{\beta}\right)$ generated by representations where $S^{1}$ acts by scalar multiplication.

To understand the group $R_{\beta}(G)$ we will restrict it to all the cyclic subgroups of $G$; let's do this by choosing a generator on each cyclic subgroup. Then, for $g$ in $G$ and $C=\langle g\rangle$ the cyclic group it generates, by restriction we have a map $R_{\beta}(G) \rightarrow$ $R_{\text {res }(\beta)}(\langle g\rangle)$ where $\operatorname{res}(\beta)$ is the restriction of $\beta$ to the group $\langle g\rangle$. As $H^{2}\left(\langle g\rangle ; S^{1}\right)=0$ we have that $\operatorname{res}(\beta)$ is cohomologous to zero, and therefore $R_{\text {res }(\beta)}(\langle g\rangle) \cong R(\langle g\rangle)$ is the untwisted representation ring of $\langle g\rangle$.

Now, these restrictions to the cyclic subgroups are endowed with an action of the group $G$; for this we need to make use of the inverse transgression of the cocycle $\beta$. The inverse transgression of $\beta$ for $h \in G$ is a 1-cocycle, and therefore is given by a homomorphism $\tau_{h} \beta: G \rightarrow S^{1}$, where $g \mapsto \beta(h, g) \beta(g, h)^{-1}$ (see [LU02, Lemma 6.4.1]). Note that these homomorphisms also have the property that $\tau_{h}(g) \cdot \tau_{k}(g)=\tau_{h k}(g)$.

Having the inverse transgression map in mind, we can now show the natural action of $G$ on $R_{r e s(\beta)}(\langle g\rangle)$. So, given a representation as

$$
\rho: \widetilde{\langle g\rangle}_{\beta} \rightarrow \operatorname{Aut}(V)
$$

for $(x, b) \in \widetilde{\langle g\rangle_{\beta}}$ and $z \in G$ we can define the representation as

$$
[z \cdot \rho]: \widetilde{\langle g\rangle}_{\beta} \rightarrow \operatorname{Aut}(V),
$$

such that

$$
[z \cdot \rho](x, b):=\rho\left((z, 1)(x, b)(z, 1)^{-1}\right) .
$$

Note that this representation has the property that

$$
[z \cdot \rho](x, b)=\tau_{x}(z) \rho(x, b),
$$

and therefore as $\tau_{x}$ is a homomorphism one has that $\left[z_{2} \cdot\left[z_{1} \cdot \rho\right]\right]=\left[z_{1} z_{2} \cdot \rho\right]$. This shows that there is a natural action of $G$ on $R_{\text {res }(\beta)}(\langle g\rangle)$.

We have that the class $\operatorname{res}(\beta)$ is trivial in cohomology; therefore there is a function $\gamma: G \rightarrow S^{1}$ such that $\delta \gamma=\beta$. Then we can make a $\operatorname{res}(\beta)$-twisted representation of $\langle g\rangle$ into a representation of $\langle g\rangle$ in the following way. A $\operatorname{res}(\beta)$ twisted representation of $\langle g\rangle$ is given by a function $\psi:\langle g\rangle \rightarrow \operatorname{Aut}(V)$ where $\psi(h k)=[\operatorname{res}(\beta)(h, k)] \psi(h) \psi(k)$. But as $\operatorname{res}(\beta)(h, k)=\gamma(k) \gamma(h k)^{-1} \gamma(h)$ we have 
that $\gamma(h k) \psi(h k)=\gamma(h) \psi(h) \gamma(k) \psi(k)$, and therefore $\gamma(\cdot) \psi(\cdot)$ becomes a $\langle g\rangle$ representation and we get the isomorphism

$$
A_{\beta, g}: R_{\text {res }(\beta)}(\langle g\rangle) \cong R(\langle g\rangle) ; \quad \psi \mapsto \gamma \psi .
$$

Now take $\zeta$ to be a $|g|$-primitive root of unity, $\mathbb{Q}(\zeta)$ the cylotomic field it generates and

$$
\chi_{g}: R(\langle g\rangle) \rightarrow \mathbb{Q}(\zeta)
$$

the trace map on the element $g$, i.e. $\chi_{g} \rho:=\operatorname{Trace}(\rho(g))$. Endow the group $R(\langle g\rangle)$ with the $G$ action coming from the isomorphism $A_{\beta, g}$ and the cyclotomic field $\mathbb{Q}(\zeta)$ with the $G$ action given by multiplication with $\tau_{g} \beta$, i.e. for $h \in G$ and $p(\zeta) \in \mathbb{Q}(\zeta)$ let $h \cdot p(\zeta)=\tau_{g} \beta(h) p(\zeta)$. In view of equation (3.4) the maps $A_{\beta, g}$ and $\chi_{g}$ become $G$-equivariant.

As the $G$ action on $R(\langle g\rangle)$ and $\mathbb{Q}(\zeta)$ depends on $\beta$, we will label these groups by $R(\langle g\rangle)_{\beta}$ and $\mathbb{Q}(\zeta)_{\beta}$; the subindex $\beta$ keeps track of how the $G$ action is defined. Let's now show the result on which the calculation of the stringy product is based.

Lemma 3.5. Let $\beta_{1}, \beta_{2}: G \times G \rightarrow S^{1}$ be two 2-cocycles representing two twisting classes. Then the diagram

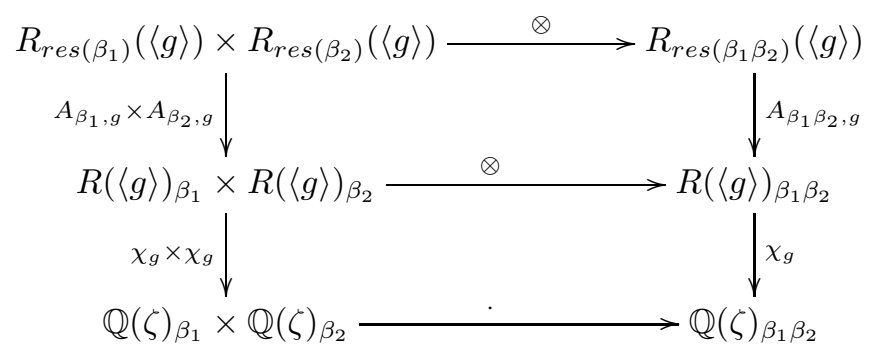

is commutative and $G$-equivariant.

Proof. Let $\psi_{i}:\langle g\rangle \rightarrow \operatorname{Aut}\left(V_{i}\right)$ be an element in $R_{\text {res }\left(\beta_{i}\right)}(\langle g\rangle)$ and let $\gamma_{i}: G \rightarrow S^{1}$ be the function such that $\delta \gamma_{i}=\beta_{i}$. We have that $A_{\beta_{i}, g}\left(\psi_{i}\right)=\gamma_{i} \psi_{i}$, and therefore

$$
A_{\beta_{1}, g}\left(\psi_{1}\right) \otimes A_{\beta_{2}, g}\left(\psi_{2}\right)=\gamma_{1} \psi_{1} \otimes \gamma_{2} \psi_{2}=\gamma_{1} \gamma_{2}\left(\psi_{1} \otimes \psi_{2}\right)=A_{\beta_{1} \beta_{2}, g}\left(\psi_{1} \otimes \psi_{2}\right),
$$

where the last equality follows from the fact that $\delta\left(\gamma_{1} \gamma_{2}\right)=\beta_{1} \beta_{2}$.

The commutativity of the second diagram follows from the fact that

$$
\operatorname{Trace}(A \otimes B)=\operatorname{Trace}(A) \operatorname{Trace}(B) \text {. }
$$

Now, for $h \in G$ we have that

$$
\left(h \cdot \rho_{1}\right) \otimes\left(h \cdot \rho_{2}\right)=\tau_{g} \beta_{1}(h) \rho_{1} \otimes \tau_{g} \beta_{2}(h) \rho_{2}=\tau_{g} \beta_{1} \beta_{2}(h) \rho_{1} \otimes \rho_{2}=h \cdot\left(\rho_{1} \otimes \rho_{2}\right),
$$

where the middle equality follows from the fact that $\tau_{g}$ is a homomorphism. This implies the equivariantness for the three horizontal lines.

The previous lemma shows how the restricted twisted representations behave once they are tensored, but we still need to show what is the appropriate decomposition for the twisted K-theory on which we can use Lemma 3.5

Let $X$ be a $G$-CW complex and $\beta: G \times G \rightarrow S^{1}$ a 2-cocycle representing the twisting class. Denote $X^{g}$ the fixed point set of $g$ and consider the following homomorphisms:

$$
{ }^{\beta} K_{G}^{*}(X) \rightarrow{ }^{r e s(\beta)} K_{\langle g\rangle}^{*}\left(X^{g}\right) \stackrel{\cong}{\rightarrow} K^{*}\left(X^{g}\right) \otimes R_{r e s(\beta)}(\langle g\rangle) \rightarrow K^{*}\left(X^{g}\right) \otimes \mathbb{Q}(\zeta)_{\beta},
$$


where the first one is the restriction map, the second is the natural isomorphism given by the trivial action of $g$ in $X^{g}$ and the last one is given by the composition $\chi_{g}$ 。 $A_{\beta, g}$. The composition of these homomorphisms has image in the invariants under the $G$ action. Then putting all these maps together for all the cyclic subgroups of $G$ we get the following result

Theorem 3.6 (Decomposition for twisted K-theory). Let $X$ be a $G$-CW complex, $G$ a finite group and $\beta: G \times G \rightarrow S^{1}$ a 2-cocycle. Let $T$ be a subset of $G$ such that any cyclic group of $G$ is generated by only one element in $T$. Then we have a decomposition

$$
{ }^{\beta} K_{G}^{*}(X) \otimes \mathbb{Q} \cong \prod_{g \in T}\left(K^{*}\left(X^{g}\right) \otimes \mathbb{Q}\left(\zeta_{|g|}\right)_{\beta}\right)^{G},
$$

where $\zeta_{|g|}$ is a primitive $|g|$-th root of unity.

Proof. The isomorphism follows directly from the decomposition formula of Adem and Ruan AR03, Theorem 7.4].

Once checks first that it holds for $X=G / H$, and then one proceeds by induction on the number of $G$ cells in $X$ with the use of the the Mayer-Vietoris exact sequence.

In view of Lemma 3.5 we have that

Theorem 3.7. The following diagram is commutative:

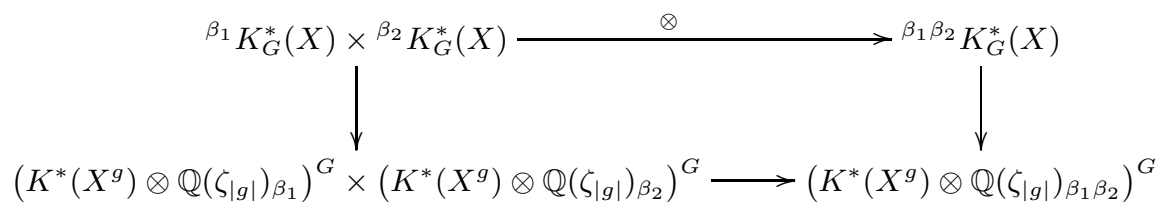

where the application of the bottom line takes the pair $\left(E_{1} \otimes p_{1}(\zeta), E_{2} \otimes p_{2}(\zeta)\right)$ and maps it to $\left(E_{1} \otimes E_{2}\right) \otimes\left(p_{1}(\zeta) p_{2}(\zeta)\right)$.

Therefore the stringy product in twisted orbifold K-theory can be calculated with the use of the decomposition formula of Theorem 3.6 and the fact that the stringy product structure decomposes as is shown in Theorem 3.7 .

Corollary 3.8. In the case that the twistings $\beta_{1}$ and $\beta_{2}$ are zero, we recover the ring isomorphism of [AR03, Thm 5.1],

$$
K_{G}^{*}(X) \otimes \mathbb{Q} \cong \prod_{g \in T}\left(K^{*}\left(X^{g}\right) \otimes \mathbb{Q}\left(\zeta_{|g|}\right)\right)^{G} .
$$

3.4. Examples. We finish this chapter by showing how the decomposition formula of Theorem 3.6 is suited for calculating the stringy product in twisted orbifold Ktheory. Let $G=(\mathbb{Z} / 2)^{3}$ and $\alpha \in H^{4}(G, \mathbb{Z})$ as in formula (3.2); we will calculate ${ }^{\alpha} K_{s t}^{*}([* / G])$ and ${ }^{\alpha} K_{s t}^{*}\left(\left[T^{6} / G\right]\right)$.

Let us remark that the ring ${ }^{\alpha} K_{s t}^{*}([* / G])$ has already been calculated by A. N. Duman in Dum without tensoring with $\mathbb{Q}$ by finding out the twisted representations and their products. We include here the calculation of this ring to make use of our decomposition formula and to clarify the calculation of ${ }^{\alpha} K_{s t}^{*}\left(\left[T^{6} / G\right]\right)$. 
Example 3.9. ${ }^{\alpha} K_{s t}^{*}([* / G])$.

As a group we have that

$$
{ }^{\alpha} K_{s t}^{*}([* / G])=\bigoplus_{g \in G}{ }^{\alpha_{g}} K_{G}^{*}(p t)=\bigoplus_{g \in G} R_{\alpha_{g}}(G),
$$

where $\alpha_{g}$ is the inverse transgression of $\alpha$ in $H^{3}(G, \mathbb{Z})$ defined in formula (3.3).

By the decomposition formula we have that

$$
R_{\alpha_{g}}(G) \otimes \mathbb{Q} \cong \prod_{h \in G}\left(\mathbb{Q}\left(\zeta_{|h|}\right)_{\alpha_{g}}\right)^{G}=\prod_{h \in G}\left(\mathbb{Q}_{\alpha_{g}}\right)^{G},
$$

where $T=G$ as all the elements generate a different cyclic group and $\mathbb{Q}\left(\zeta_{|h|}\right)=\mathbb{Q}$ because $\zeta_{|h|}$ is either 1 or -1 .

Recall that the action of $k$ in $\mathbb{Q}\left(\zeta_{|h|}\right)_{\alpha_{g}}$, for $k \in G$, is given by multiplication by $\tau_{h} \alpha_{g}(k)$, the inverse transgression of $\alpha_{g}$ evaluated in $k$. Therefore, by Lemma 3.4 we have that

$$
V_{g, h}=:\left(\mathbb{Q}\left(\zeta_{|h|}\right)_{\alpha_{g}}\right)^{G}= \begin{cases}\mathbb{Q} & \text { for } g=h, \text { or } g=0 \text { or } h=0, \\ 0 & \text { otherwise. }\end{cases}
$$

Therefore we have that for $g \neq 0$

$$
R_{\alpha_{g}}(G) \otimes \mathbb{Q} \cong V_{g, 1} \times V_{g, g} \cong \mathbb{Q} \times \mathbb{Q}
$$

and as $\alpha_{1}=\tau_{1} \alpha=0$ we have that

$$
R_{\alpha_{1}}(G) \otimes \mathbb{Q}=R(G) \otimes \mathbb{Q} \cong \prod_{h \in G} \mathbb{Q},
$$

where this last isomorphism is an isomorphism of rings. Therefore we have that the rank of ${ }^{\alpha} K_{s t}^{*}([* / G])$ is $2 \times 7+8=22$. We point out here that the rank of this ring was already calculated in ARZ, Example 6.2].

The product structure is easy to determine following Theorem 3.7, Let's denote by $1_{g, h} \in \prod_{h \in G} V_{g, h}$ the element which is 1 in the component of $h$ and 0 on the other components. Then we have that

$$
1_{g_{1}, h_{1}} \cdot 1_{g_{2}, h_{2}}=\left\{\begin{array}{cl}
1_{1, h_{1}} & \text { if } 0=g_{1} g_{2} \text { and } h_{1}=h_{2}, \\
1_{g_{1} g_{2}, h_{1}} & \text { if } h_{1}=h_{2}=g_{1} g_{2} \neq 0, \\
0 & \text { otherwise. }
\end{array}\right.
$$

We finish this example by noting that the ring generated by $\bigoplus_{g \in G} V_{g, 1}$ is isomorphic to the group ring $\mathbb{Q}[G]$. Therefore we have that both the group ring $\mathbb{Q}[G]$ and the representation $\operatorname{ring} R(G) \otimes \mathbb{Q}$ are subrings of ${ }^{\alpha} K_{s t}^{*}([* / G])$; the last one because $R_{\alpha_{1}}(G)=R(G)$.

Example 3.10. ${ }^{\alpha} K_{s t}^{*}\left(\left[T^{6} / G\right]\right)$.

Let's consider the torus $T^{2}=\mathbb{C} /(\mathbb{Z} \oplus \mathbb{Z})$ together with the $\mathbb{Z} / 2$ action given by multiplication by -1 . The action fixes four points $\left\{0, \frac{1}{2}, \frac{1}{2} i, \frac{1}{2}+\frac{1}{2} i\right\}$, and we will denote this set by $4 *$. The group $\mathbb{Z} / 2$ acts on $K^{*}\left(T^{2}\right)$ by the trivial action on $K^{0}\left(T^{2}\right)$ and by the sign action on $K^{1}\left(T^{2}\right)$.

Then we can consider the group $G=(\mathbb{Z} / 2)^{3}$ acting coordinate-wise on $M=$ $T^{6}=T^{2} \times T^{2} \times T^{2}$; that is, for $g=\left(a_{1}, a_{2}, a_{3}\right) \in G$ and $z=\left(z_{1}, z_{2}, z_{3}\right) \in T^{6}$, the action is $g \cdot z:=\left((-1)^{a_{1}} z_{1},(-1)^{a_{2}} z_{2},(-1)^{a_{3}} z_{3}\right)$.

In what follows we will explicitly calculate the rank of ${ }^{\alpha} K_{s t}^{*}\left(\left[T^{6} / G\right]\right)$ and we will show how the ring structure behaves for some chosen elements. We will not 
calculate how the ring structure behaves for all the cases because the rank of the twisted orbifold K-theory is too big.

Let's start by calculating the rank of ${ }^{\alpha} K_{s t}^{*}\left(\left[T^{6} / G\right]\right)$.

For $g \in G$, by Theorem 3.6 we have that

$$
{ }^{\alpha_{g}} K_{G}^{*}\left(M^{g}\right) \otimes \mathbb{Q} \cong \prod_{h \in G}\left(K^{*}\left(M^{g, h}\right) \otimes \mathbb{Q}_{h, \alpha_{g}}\right)^{G},
$$

where $G$ acts on $\mathbb{Q}_{h, \alpha_{g}}=\mathbb{Q}$ multiplying by the double inverse transgression $\tau_{h} \tau_{g} \alpha=$ $\tau_{h} \alpha_{g}$. Therefore for the elements $g \in\{(0,0,0),(1,0,0),(1,1,0),(1,1,1)\}$ we will elaborate some tables where we will calculate the double inverse transgressions $\tau_{h} \tau_{g} \alpha$, the fixed points $M^{g, h}$, and the invariant set $\left(K^{*}\left(M^{g, h}\right) \otimes \mathbb{Q}_{h, \alpha_{g}}\right)^{G}$ together with its rank.

\begin{tabular}{|c|c|c|c|c|}
\hline \multicolumn{5}{|c|}{$\bar{\alpha}_{g} K_{G}^{*}\left(M^{g}\right) \otimes \mathbb{Q} \quad$ for $g=(1,0,0)$} \\
\hline$h$ & $\tau_{h} \tau_{g} \alpha$ & $M^{g, h}$ & $\left(K\left(M^{g, h}\right) \otimes \mathbb{Q}_{h, \alpha_{g}}\right)^{G}$ & rank \\
\hline$(0,0,0)$ & 0 & $4 * \times T^{2} \times T^{2}$ & $K^{0}(4 *) \otimes K^{0}\left(T^{2}\right) \otimes K^{0}\left(T^{2}\right)$ & 16 \\
\hline$(1,0,0)$ & 0 & $4 * \times T^{2} \times T^{2}$ & $K^{0}(4 *) \otimes K^{0}\left(T^{2}\right) \otimes K^{0}\left(T^{2}\right)$ & 16 \\
\hline$(0,1,0)$ & $x_{3}^{2}$ & $4 * \times 4 * \times T^{2}$ & $K^{0}(4 *) \otimes K^{0}(4 *) \otimes K^{1}\left(T^{2}\right)$ & 32 \\
\hline$(0,0,1)$ & $x_{2}^{2}$ & $4 * \times T^{2} \times 4 *$ & $K^{0}(4 *) \otimes K^{1}\left(T^{2}\right) \otimes K^{0}(4 *)$ & 32 \\
\hline$(1,1,0)$ & $x_{3}^{2}$ & $4 * \times 4 * \times T^{2}$ & $K^{0}(4 *) \otimes K^{0}(4 *) \otimes K^{1}\left(T^{2}\right)$ & 32 \\
\hline$(0,1,1)$ & $x_{2}^{2}+x_{3}^{2}$ & $4 * \times 4 * \times 4 *$ & 0 & 0 \\
\hline$(1,0,1)$ & $x_{2}^{2}$ & $4 * \times T^{2} \times 4 *$ & $K^{0}(4 *) \otimes K^{1}\left(T^{2}\right) \times K^{0}(4 *)$ & 32 \\
\hline$(1,1,1)$ & $x_{2}^{2}+x_{3}^{2}$ & $4 * \times 4 * \times 4 *$ & 0 & \\
\hline & & & Total rank & 160 \\
\hline
\end{tabular}

\begin{tabular}{|c|c|c|c|c|}
\hline \multicolumn{5}{|c|}{$\bar{\alpha}_{g} K_{G}^{*}\left(M^{g}\right) \otimes \mathbb{Q} \quad$ for $g=(1,1,0)$} \\
\hline$h$ & $\tau_{h} \tau_{g} \alpha$ & $M^{g, h}$ & $\left(K\left(M^{g, h}\right) \otimes \mathbb{Q}_{h, \alpha_{g}}\right)^{G}$ & rank \\
\hline$(0,0,0)$ & 0 & $4 * \times 4 * \times T^{2}$ & $K^{0}(4 *) \otimes K^{0}(4 *) \otimes K^{0}\left(T^{2}\right)$ & 32 \\
\hline$(1,0,0)$ & $x_{3}^{2}$ & $4 * \times 4 * \times T^{2}$ & $K^{0}(4 *) \otimes K^{0}(4 *) \otimes K^{1}\left(T^{2}\right)$ & 32 \\
\hline$(0,1,0)$ & $x_{3}^{2}$ & $4 * \times 4 * \times T^{2}$ & $K^{0}(4 *) \otimes K^{0}(4 *) \otimes K^{1}\left(T^{2}\right)$ & 32 \\
\hline$(0,0,1)$ & $x_{1}^{2}+x_{2}^{2}$ & $4 * \times 4 * \times 4 *$ & 0 & 0 \\
\hline$(1,1,0)$ & 0 & $4 * \times 4 * \times T^{2}$ & $K^{0}(4 *) \otimes K^{0}(4 *) \otimes K^{0}\left(T^{2}\right)$ & 32 \\
\hline$(0,1,1)$ & $x_{1}^{2}+x_{2}^{2}+x_{3}^{2}$ & $4 * \times 4 * \times 4 *$ & 0 & 0 \\
\hline$(1,0,1)$ & $x_{1}^{2}+x_{2}^{2}+x_{3}^{2}$ & $4 * \times 4 * \times 4 *$ & 0 & 0 \\
\hline$(1,1,1)$ & $x_{1}^{2}+x_{2}^{2}$ & $4 * \times 4 * \times 4 *$ & 0 & 0 \\
\hline & & & & 128 \\
\hline
\end{tabular}

\begin{tabular}{|c|c|c|c|c|}
\hline \multicolumn{5}{|c|}{$\bar{\alpha}_{g} K_{G}^{*}\left(M^{g}\right) \otimes \mathbb{Q} \quad$ for $g=(1,1,1)$} \\
\hline$h$ & $\tau_{h} \tau_{g} \alpha$ & $M^{g, h}$ & $\left(K\left(M^{g, h}\right) \otimes \mathbb{Q}_{h, \alpha_{g}}\right)^{G}$ & rank \\
\hline$(0,0,0)$ & 0 & $4 * \times 4 * \times 4 *$ & $K^{0}(4 *) \otimes K^{0}(4 *) \otimes K^{0}(4 *)$ & 64 \\
\hline$(1,0,0)$ & $x_{2}^{2}+x_{3}^{2}$ & $4 * \times 4 * \times 4 *$ & 0 & 0 \\
\hline$(0,1,0)$ & $x_{1}^{2}+x_{3}^{2}$ & $4 * \times 4 * \times 4 *$ & 0 & 0 \\
\hline$(0,0,1)$ & $x_{1}^{2}+x_{2}^{2}$ & $4 * \times 4 * \times 4 *$ & 0 & 0 \\
\hline$(1,1,0)$ & $x_{1}^{2}+x_{2}^{2}$ & $4 * \times 4 * \times 4 *$ & 0 & 0 \\
\hline$(0,1,1)$ & $x_{2}^{2}+x_{3}^{\overline{2}}$ & $4 * \times 4 * \times 4 *$ & 0 & 0 \\
\hline$(1,0,1)$ & $x_{1}^{2}+x_{3}^{2}$ & $4 * \times 4 * \times 4 *$ & 0 & 0 \\
\hline$(1,1,1)$ & $0^{5}$ & $4 * \times 4 * \times 4 *$ & $K^{0}(4 *) \otimes K^{0}(4 *) \otimes K^{0}(4 *)$ & 64 \\
\hline & & & Total rank & 128 \\
\hline
\end{tabular}


For $g=(0,0,0)$ the inverse transgression $\tau_{g} \alpha=0$ and therefore we just need to calculate the invariants for each $h$

\begin{tabular}{|c|c|c|c|}
\hline \multicolumn{4}{|c|}{$K_{G}^{*}\left(M^{g}\right) \otimes \mathbb{Q} \quad$ for $g=(0,0,0)$} \\
\hline$h$ & $M^{g, h}$ & $\left(K\left(M^{g, h}\right) \otimes \mathbb{Q}\right)^{G}$ & rank \\
\hline$(0,0,0)$ & $\overline{T^{2} \times T^{2} \times T^{2}}$ & $K^{0}\left(T^{2}\right) \otimes K^{0}\left(T^{2}\right) \otimes K^{0}\left(T^{2}\right)$ & 8 \\
\hline$(1,0,0)$ & $4 * \times T^{2} \times T^{2}$ & $K^{0}(4 *) \otimes K^{0}\left(T^{2}\right) \otimes K^{0}\left(T^{2}\right)$ & 16 \\
\hline$(0,1,0)$ & $T^{2} \times 4 * \times T^{2}$ & $K^{0}\left(T^{2}\right) \otimes K^{0}(4 *) \otimes K^{0}\left(T^{2}\right)$ & 16 \\
\hline$(0,0,1)$ & $T^{2} \times T^{2} \times 4 *$ & $K^{0}\left(T^{2}\right) \otimes K^{0}\left(T^{2}\right) \otimes K^{0}(4 *)$ & 16 \\
\hline$(1,1,0)$ & $4 * \times 4 * \times T^{2}$ & $K^{0}(4 *) \otimes K^{0}(4 *) \otimes K^{0}\left(T^{2}\right)$ & 32 \\
\hline$(0,1,1)$ & $T^{2} \times 4 * \times 4 *$ & $K^{0}\left(T^{2}\right) \otimes K^{0}(4 *) \otimes K^{0}(4 *)$ & 32 \\
\hline$(1,0,1)$ & $4 * \times T^{2} \times 4 *$ & $K^{0}(4 *) \otimes K^{0}\left(T^{2}\right) \times K^{0}(4 *)$ & 32 \\
\hline$(1,1,1)$ & $4 * \times 4 * \times 4 *$ & $K^{0}(4 *) \otimes K^{1}(4 *) \otimes K^{0}(4 *)$ & 64 \\
\hline & & Total rank & 216 \\
\hline
\end{tabular}

As the groups ${ }^{\alpha_{g}} K_{G}^{*}\left(M^{g}\right)$ are isomorphic for $g \in\{(1,0,0),(0,1,0),(0,0,1)\}$ and for $g \in\{(1,1,0),(1,0,1),(0,1,1)\}$, we can conclude that

$$
\operatorname{rank}^{\alpha} K_{s t}^{*}\left(\left[T^{6} / G\right]\right)=216+3 \times 160+3 \times 128+128=1208 .
$$

The stringy product now is easy to calculate. We just need to find the obstruction bundle and the pushforward maps.

First note that all the obstruction bundles are trivial. This is because the eigenvalues of the action of any element in $G$ is 1 or -1 , and therefore the $a_{j}+b_{j}$ of Definition 2.2 are always smaller than or equal to 1 .

The pushforwards of the stringy product can be calculated by understanding the $\mathbb{Z} / 2$-equivariant pushforward of the inclusion $4 * \rightarrow T^{2}$. Let's denote by $\eta \in K_{\mathbb{Z} / 2}^{0}\left(T^{2}\right)$ the trivial one dimensional bundle whose action of $\mathbb{Z} / 2$ is given by multiplication by -1 composed with the geometrical action, and let $H \in K_{\mathbb{Z} / 2}^{0}\left(T^{2}\right)$ be the pullback of the Hopf bundle under the degree 1 map $T^{2} \rightarrow S^{2}$ together with the pullback action of $\mathbb{Z} / 2$. If we consider only one point $i: * \rightarrow T^{2}$, the pushforward map is

$$
i_{!}: R(\mathbb{Z} / 2) \rightarrow K_{\mathbb{Z} / 2}^{0}\left(T^{2}\right), \quad i_{!}(1)=1-H \otimes \eta,
$$

and therefore $i^{*} i_{!}(1)=1-\xi$, where $\xi$ is the sign representation.

For $E \in{ }^{\alpha_{g}} K_{G}^{*}\left(M^{g}\right)$ and $F \in{ }^{\alpha_{k}} K_{G}^{*}\left(M^{k}\right)$, by Definition 3.1, their stringy product is

$$
E \star F:=e_{3 !}\left(e_{1}^{*} E \otimes e_{2}^{*} F\right) \in{ }^{\alpha_{g k}} K_{G}^{*}\left(M^{g k}\right) .
$$

By the decomposition formula we have that $E \mapsto \prod_{h} E_{h}$ and $F \mapsto \prod_{h} F_{h}$, and by Theorem 3.7 we have that $\left(e_{1}^{*} E \otimes e_{2}^{*} F\right)_{h} \cong e_{1}^{*} E_{h} \otimes e_{2}^{*} F_{h}$. The only part where one needs to be careful is with the behavior of the pushforward map

$$
e_{3 !}:{ }^{\alpha_{g k}} K_{G}^{*}\left(M^{g, k}\right) \rightarrow{ }^{\alpha_{g k}} K_{G}^{*}\left(M^{g k}\right),
$$

under the decomposition formula.

As there would be too many cases to consider, we will only show how to calculate the stringy product for $g=(1,0,0), k=(1,1,0)$ and $g k=(0,1,0)$. For the other cases one can imitate the following procedure. 
First let's calculate ${ }^{\alpha_{g k}} K_{G}^{*}\left(M^{g, k}\right)$ in order to know which components are nonzero in its decomposition:

\begin{tabular}{|c|c|c|c|}
\hline \multicolumn{4}{|c|}{$\alpha_{g k} K_{G}\left(M^{g, k}\right) \otimes \mathbb{Q} \quad$ for $g=(1,0,0)$ and $k=(1,1,0)$} \\
\hline$h$ & $\tau_{h} \tau_{g k} \alpha$ & $M^{g, k, h}$ & $\left(K\left(M^{g, k, h}\right) \otimes \mathbb{Q}_{h, \alpha_{g k}}\right)^{G}$ \\
\hline$\overline{(0,0,0)}$ & 0 & $4 * \times 4 * \times T^{2}$ & $K^{0}(4 *) \otimes K^{0}(4 *) \otimes K^{0}\left(T^{2}\right)$ \\
\hline$(1,0,0)$ & $x_{3}^{2}$ & $4 * \times 4 * \times T^{2}$ & $K^{0}(4 *) \otimes K^{0}(4 *) \otimes K^{1}\left(T^{2}\right)$ \\
\hline$(0,1,0)$ & 0 & $4 * \times 4 * \times T^{2}$ & $K^{0}(4 *) \otimes K^{0}(4 *) \otimes K^{0}\left(T^{2}\right)$ \\
\hline$(0$, & $x_{1}^{2}$ & $4 * \times 4 * \times 4 *$ & 0 \\
\hline$(1,1,0)$ & $x_{3}^{2}$ & $4 * \times 4 * \times T^{2}$ & $K^{0}(4 *) \otimes K^{0}(4 *) \otimes K^{1}\left(T^{2}\right)$ \\
\hline$(0,1,1)$ & $x_{1}^{2}$ & $4 * \times 4 * \times 4 *$ & 0 \\
\hline$(1,0,1)$ & $x_{1}^{2}+x_{3}^{2}$ & $4 * \times 4 * \times 4 *$ & 0 \\
\hline$(1,1,1)$ & $x_{1}^{2}+x_{3}^{2}$ & $4 * \times 4 * \times 4 *$ & 0 \\
\hline
\end{tabular}

Now let's see how the pushforward

$$
{ }^{\alpha_{g k}} K_{G}^{*}\left(M^{g, k}\right) \longrightarrow e_{3 !}{ }^{\alpha_{g k}} K_{G}^{*}\left(M^{g k}\right)
$$

behaves on each of the non-zero components (on the left we have $\left(K^{*}\left(M^{g, k, h}\right) \otimes\right.$ $\left.\mathbb{Q}_{h, \alpha_{g k}}\right)^{G}$ and on the right $\left.\left(K^{*}\left(M^{g k, h}\right) \otimes \mathbb{Q}_{h, \alpha_{g k}}\right)^{G}\right)$.

For $h=(0,0,0)$

$$
K^{0}(4 *) \otimes K^{0}(4 *) \otimes K^{0}\left(T^{2}\right) \stackrel{(1-H) \otimes 1 \otimes 1}{\longrightarrow} K^{0}\left(T^{2}\right) \otimes K^{0}(4 *) \otimes K^{0}\left(T^{2}\right),
$$

where the map is given by multiplying with the element $(1-H) \otimes 1 \otimes 1$ (the Thom class of the inclusion).

For $h=(1,0,0)$

$$
K^{0}(4 *) \otimes K^{0}(4 *) \otimes K^{0}\left(T^{2}\right) \stackrel{2 \otimes 1 \otimes 1}{\longrightarrow} K^{0}(4 *) \otimes K^{0}(4 *) \otimes K^{0}\left(T^{2}\right),
$$

because $(1-H \otimes \eta) \otimes 1 \otimes 1$ restricted to $4 * \times 4 * \times T^{2}$ is $(1-\xi) \otimes 1 \otimes 1$, and its character on $h=(1,0,0)$ is $(2 \otimes 1 \otimes 1)$.

For $h=(0,1,0)$

$$
K^{0}(4 *) \otimes K^{0}(4 *) \otimes K^{0}\left(T^{2}\right) \stackrel{(1-H) \otimes 1 \otimes 1}{\longrightarrow} K^{0}\left(T^{2}\right) \otimes K^{0}(4 *) \otimes K^{0}\left(T^{2}\right),
$$

because the character of $(1-H \otimes \eta) \otimes 1 \otimes 1$ in $h=(0,1,0)$ is $1-H \otimes 1 \otimes 1$.

For $h=(1,1,0)$

$$
K^{0}(4 *) \otimes K^{0}(4 *) \otimes K^{0}\left(T^{2}\right) \stackrel{2 \otimes 1 \otimes 1}{\longrightarrow} K^{0}(4 *) \otimes K^{0}(4 *) \otimes K^{0}\left(T^{2}\right),
$$

because the character of $(1-\xi) \otimes 1 \otimes 1$ in $h=(1,1,0)$ is $2 \otimes 1 \otimes 1$.

The decomposition for the stringy product $E \star F$ can now be easily calculated. Write $E_{h}:=\left(E_{h}^{1}, E_{h}^{2}, E_{h}^{3}\right)$ and $F_{h}=\left(F_{h}^{1}, F_{h}^{2}, F_{h}^{3}\right)$ in terms of Kunneth decomposition as in the tables above. For example, for $h=(1,1,0)$ we have that $\left(E_{h}^{1}, E_{h}^{2}, E_{h}^{3}\right) \in K^{0}(4 *) \otimes K^{0}(4 *) \otimes K^{1}\left(T^{2}\right)$. If we call the inclusion $j: 4 * \rightarrow T^{2}$, then we have that

$$
(E \star F)_{h}= \begin{cases}\left((1-H) \otimes E_{h}^{1} \otimes F_{h}^{1}\right) \otimes\left(j^{*} E_{h}^{2} \otimes F_{h}^{2}\right) \otimes\left(E_{h}^{3} \otimes F_{h}^{3}\right) & \text { for } h=(0,0,0), \\ \left.2 E_{h}^{1} \otimes F_{h}^{1}\right) \otimes\left(j^{*} E_{h}^{2} \otimes F_{h}^{2}\right) \otimes\left(E_{h}^{3} \otimes F_{h}^{3}\right) & \text { for } h=(1,0,0), \\ \left.(1-H) \otimes E_{h}^{1} \otimes F_{h}^{1}\right) \otimes\left(E_{h}^{2} \otimes F_{h}^{2}\right) \otimes\left(E_{h}^{3} \otimes F_{h}^{3}\right) & \text { for } h=(0,1,0), \\ \left(2 E_{h}^{1} \otimes F_{h}^{1}\right) \otimes\left(E_{h}^{2} \otimes F_{h}^{2}\right) \otimes\left(E_{h}^{3} \otimes F_{h}^{3}\right) & \text { for } h=(1,1,0), \\ 0 & \text { otherwise. }\end{cases}
$$




\section{ACKNOWLEDGEMENTS}

We would like to thank Professors Alejandro Adem, Shengda Hu, Ernesto Lupercio and Yongbin Ruan for their conversations. The second author would like to thank the hospitality of the Pacific Institute for the Mathematical Sciences PIMS where parts of this work were carried out. Both authors acknowledge the support of COLCIENCIAS through the grant number 120440520246.

\section{REFERENCES}

[AR03] A. Adem and Y. Ruan. Twisted orbifold K-theory. Comm. Math. Phys., 237(3):533-556, 2003. MR.1993337 (2004e:19004)

[ARZ] A. Adem, Y. Ruan, and B. Zhang. A stringy product on twisted orbifold $K$-theory. arxiv:math.AT/0605534.

[AS68] M. F. Atiyah and I. M. Singer. The index of elliptic operators. III. Ann. of Math. (2), 87:546-604, 1968. MR0236952 (38:5245)

[AS69] M. F. Atiyah and G. B. Segal. Equivariant $K$-theory and completion. J. Differential Geometry, 3:1-18, 1969. MR0259946 (41:4575)

[CH06] B. Chen and S. Hu. A deRham model for Chen-Ruan cohomology ring of abelian orbifolds. Math. Ann., 336(1):51-71, 2006. MR.2242619 (2007d:14044)

[CR04] W. Chen and Y. Ruan. A new cohomology theory of orbifold. Comm. Math. Phys., 248(1):1-31, 2004. MR2104605 (2005j:57036)

[Dum] Ali Nabi Duman. An example of a twisted fusion algebra. Preprint.

[JKK07] Tyler J. Jarvis, Ralph Kaufmann, and Takashi Kimura. Stringy $K$-theory and the Chern character. Invent. Math., 168(1):23-81, 2007. MR22285746 (2007i:14059)

[LO01] W. Lück and B. Oliver. Chern characters for the equivariant $K$-theory of proper $G$-CWcomplexes. In Cohomological methods in homotopy theory (Bellaterra, 1998), volume 196 of Progr. Math., pages 217-247. Birkhäuser, Basel, 2001. MR.1851256 (2002m:55016)

[LU02] E. Lupercio and B. Uribe. Loop groupoids, gerbes, and twisted sectors on orbifolds. In Orbifolds in mathematics and physics (Madison, WI, 2001), volume 310 of Contemp. Math., pages 163-184. Amer. Math. Soc., Providence, RI, 2002. MR.1950946 (2004c:58043)

[Moe02] I. Moerdijk. Orbifolds as groupoids: An introduction. In Orbifolds in mathematics and physics (Madison, WI, 2001), volume 310 of Contemp. Math., pages 205-222. Amer. Math. Soc., Providence, RI, 2002. MR.1950948 (2004c:22003)

[Qui71] D. Quillen. Elementary proofs of some results of cobordism theory using Steenrod operations. Advances in Math., 7:29-56 (1971), 1971. MR0290382 (44:7566)

[Seg68] G. Segal. Equivariant K-theory. Inst. Hautes Études Sci. Publ. Math., (34):129-151, 1968. MR.0234452 (38:2769)

Departamento de Matemáticas, Universidad de los Andes, Carrera 1 N. 18A - 10, Bogotá, Colombia

E-mail address: es.becerra75@uniandes.edu.co

Departamento de Matemáticas, Universidad de los Andes, Carrera 1 N. 18A - 10, Bogotá, Colombia

E-mail address: buribe@uniandes.edu.co 